OAK RIDGE

NATIONAL

LABORATORY

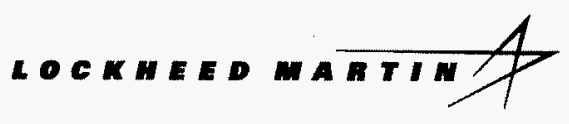

SFCEIVED

Allis 071997

OSTI

Robert N. Nodine

Roberto Lenarduzzi

\title{
Sensor Concentrator Unit for the Continuous Automated Vault Inventory System
}


This report has been reproduced directly from the best available copy.

Available to DOE and DOE contractors from the Office of Scientific and Technical Information, P. O. Box 62, Oak Ridge. TN 37831 ; prices available from (423) 576-8401. FTS 626-8401.

Available to the public from the National Technical Information Service, U.S. Department of Commerce, 5285 Port Royal Road, Springfield, VA 22161.

This report was prepared as an account of work sponsored by an agency of the United States Government. Neither the United States Government nor any agency thereof. nor any of their employees, makes any warranty, express or implied, or assumes any legal liability or responsibility for the accuracy. completeness, or usefulness of any information, apparatus, product, or process disclosed, or represents that its use would not infringe privately owned rights. Reference herein to any specific commercial product, process, or service by trade name, trademark, manufacturer, or otherwise, does not necessarily constitute or imply its endorsement, recommendation, or favoring by the United States Government or any agency thereof. The views and opinions of authors expressed herein do not necessarily state or reflect those of the United States Government of any agency thereof. 
ORNL/TM-1341 1

Instrumentation and Controls Division

\title{
SENSOR CONCENTRATOR UNIT FOR THE CONTINUOUS AUTOMATED VAULT INVENTORY SYSTEM
}

\author{
Robert N. Nodine \\ Roberto Lenarduzzi
}

Date Published-June 1997

\author{
Prepared by the \\ Oak Ridge National Laboratory \\ Oak Ridge, Tennessee 37831-6003 \\ Managed by \\ LOCKHEED MARTIN ENERGY RESEARCH CORP. \\ for the \\ U.S. DEPARTMENT OF ENERGY \\ under contract DE-AC05-96OR22464
}




\section{CONTENTS}

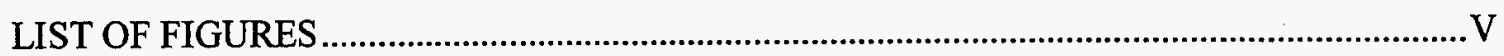

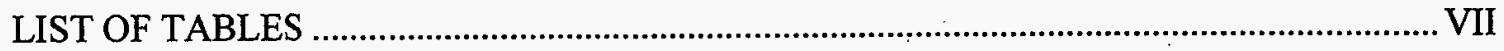

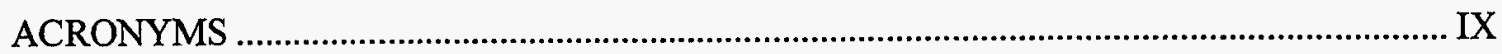

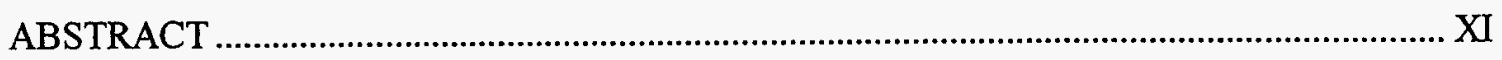

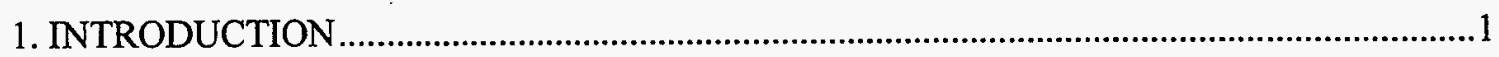

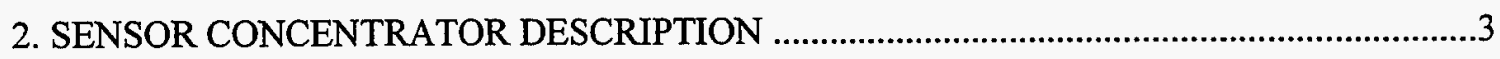

2.1. SENSOR CONCENTRATOR FUNCTIONAL BLOCKS …...........................................

2.2. SENSOR CONNECTION AND SIGNAL DISTRIBUTION ......................................6

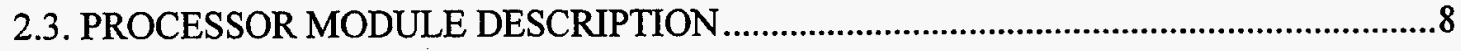

2.4. GAMMA SENSOR INTERFACE DESCRIPTION ........................................................

2.5. WEIGHT PAD SENSOR INTERFACE DESCRIPTION .............................................11

2.6. RELATIONSHIP BETWEEN SENSOR LOCATION AND DATA ...............................13

3. STANDALONE SENSOR CONCENTRATOR OPERATION …......................................15

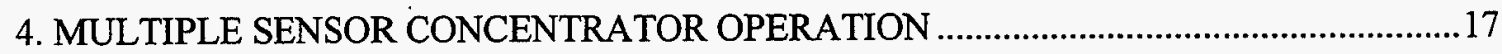

4.1. POWER AND COMMUNICATION DISTRIBUTION UNIT ........................................17

5. SENSOR MODULE INTERFACE REQUIERMENTS ........................................................19

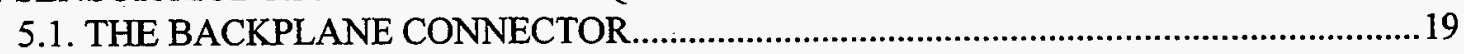

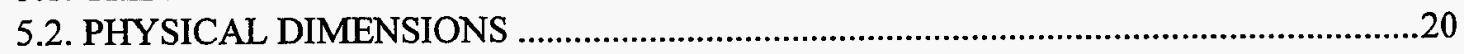

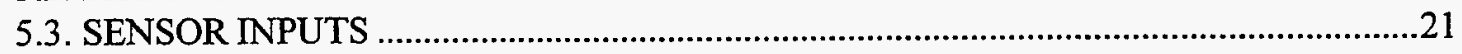

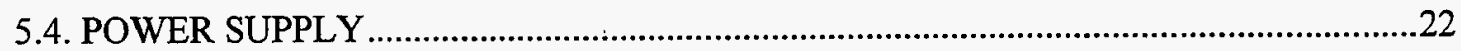

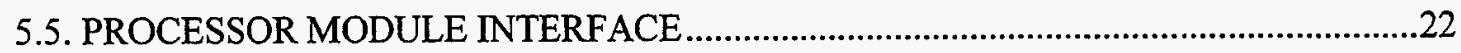

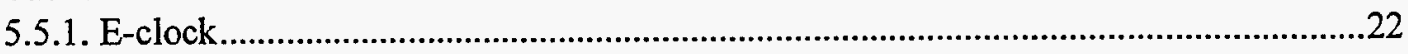

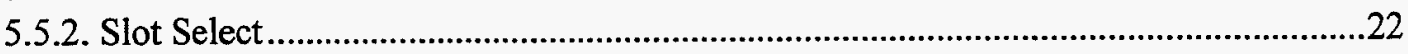

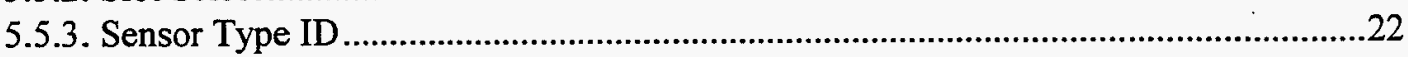

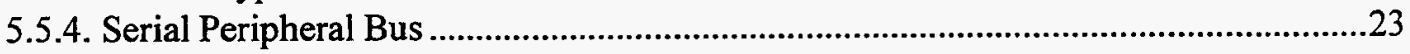

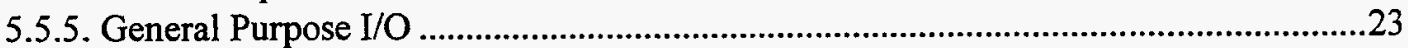

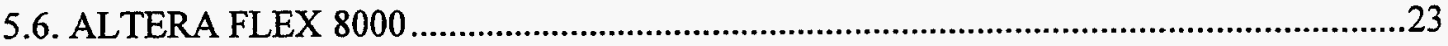

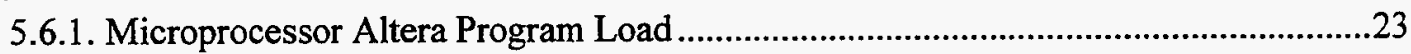

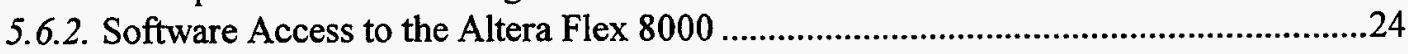

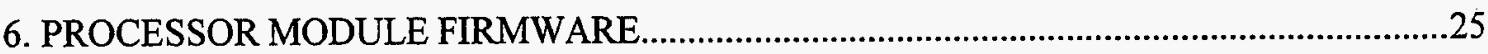

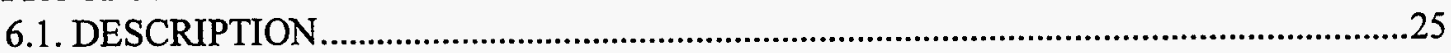

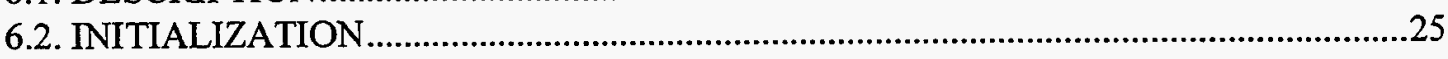

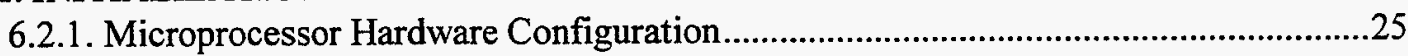

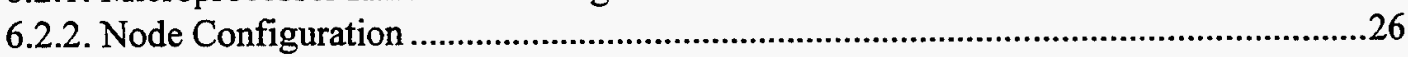

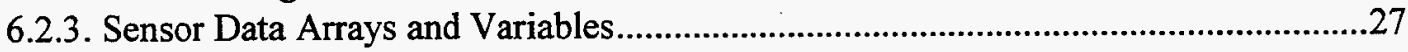

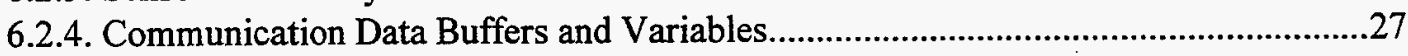

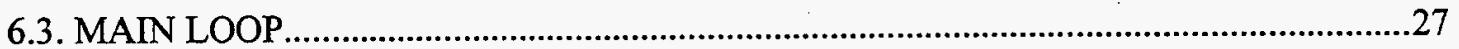




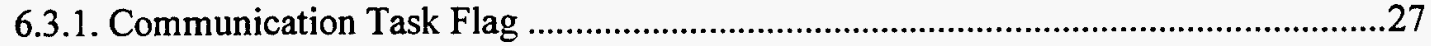

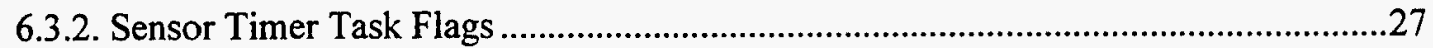

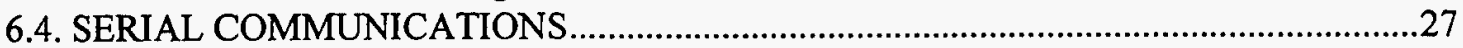

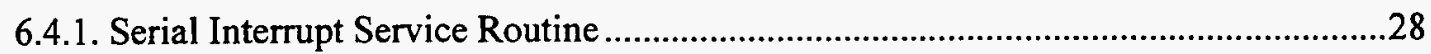

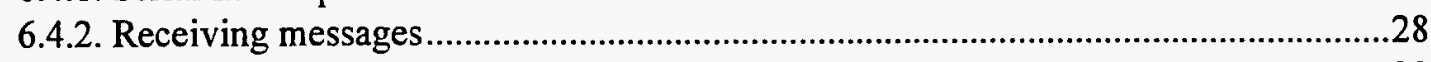

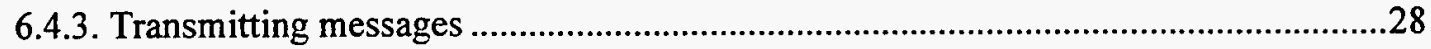

6.5. SENSOR DATA ACQUISITION

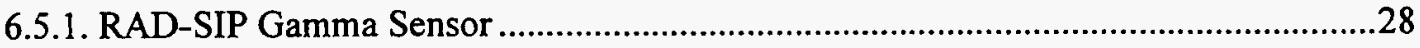

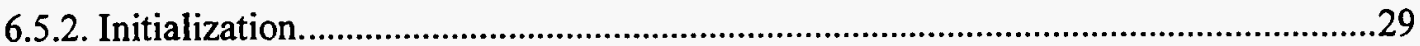

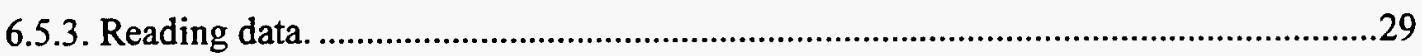

6.6. CAPACITIVE WEIGHT PAD AND TEMPERATURE SENSOR ................................29

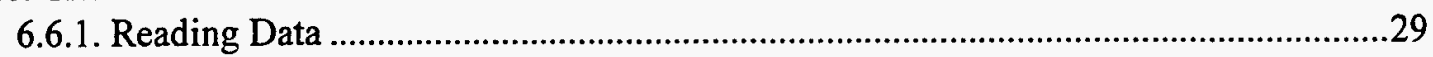

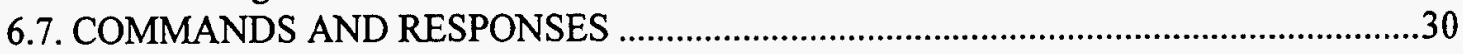

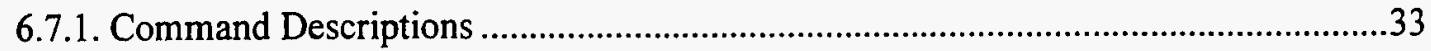

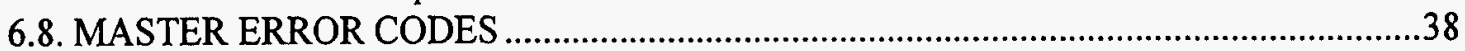

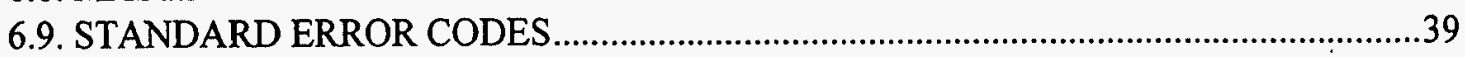

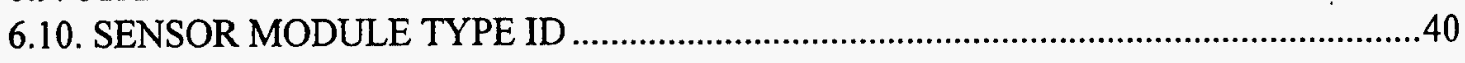

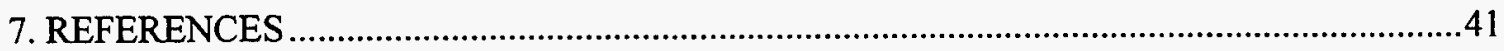

Appendix A: CAVIS SENSOR BUS PROTOCOL AND COMMANDS ..................................42 


\section{LIST OF FIGURES}

Page

Fig. 1. Bottom view of the sensor concentrator. ....................................................................

Fig. 2. Inside view of the sensor concentrator with three module removed....................................

Fig. 3 Sensor concentrator functional block diagram. ....................................................................

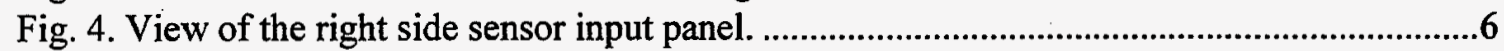

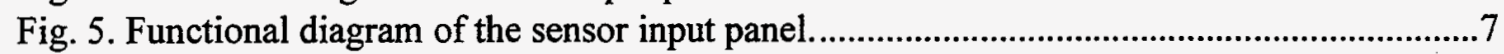

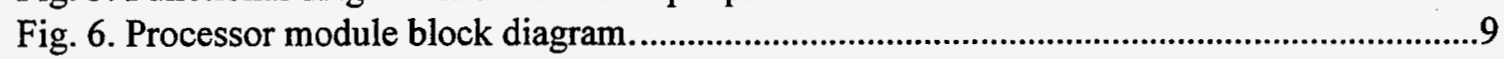

Fig. 7. Functional diagram of RAD-SIP sensor module ...........................................................

Fig. 8. Functional diagram of CAP-WT sensor module. ...........................................................12

Fig. 9. Diagram of how data relate to slot position....................................................................14

Fig. 10. Photograph of a CAVIS sensor demonstration..............................................................15

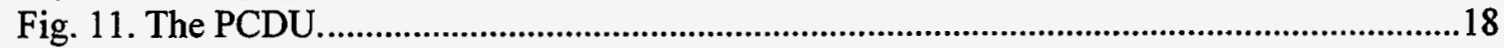

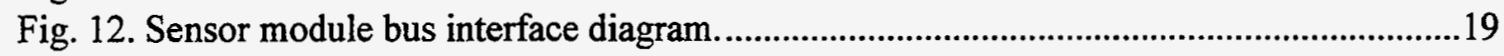

Fig. 13. Sensor module dimension requirements....................................................................21 


\section{LIST OF TABLES}

Page

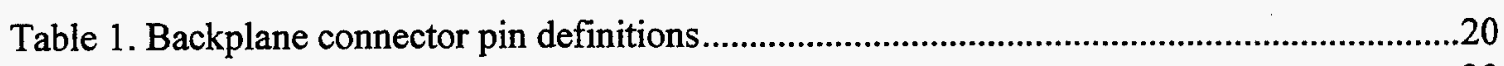

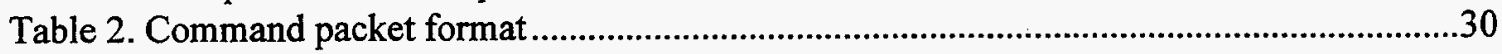

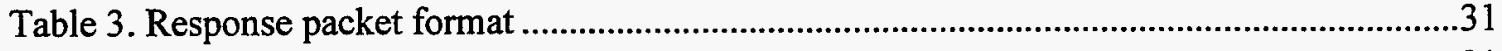

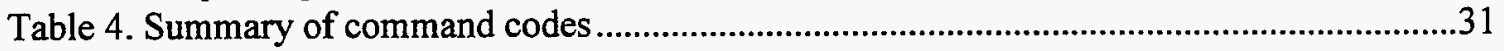

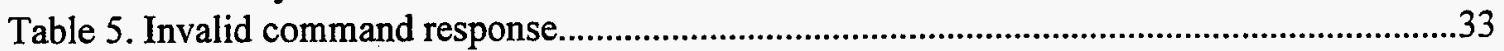

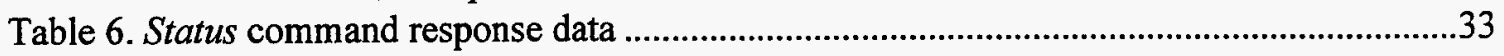

Table 7. Configuration command response data .........................................................................3

Table 8. Send Report A / Send Report B response data.................................................................34

Table 9. Read Complete Configuration command response data ...................................................35

Table 10. Read Sensor Type ID command parameters .................................................................35

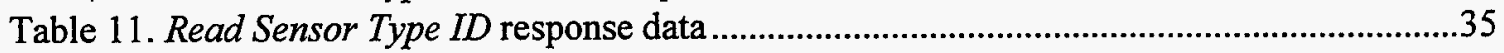

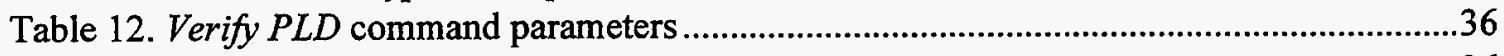

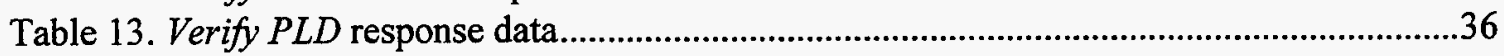

Table 14. Read Single Data Channel command parameters ......................................................36

Table 15. Read Single Data Channel response data ...................................................................36

Table 16. Reinitialize ALL command response data .....................................................................37

Table 17. Read Node Address command parameters .....................................................................37

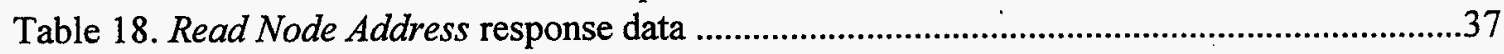

Table 19. Set Node Address command parameters ........................................................................37

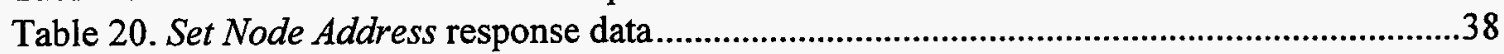

Table 21. Set processor module 48-bit Serial ID command parameters .......................................38

Table 22. Set processor module 48-bit Serial ID response data ..................................................38

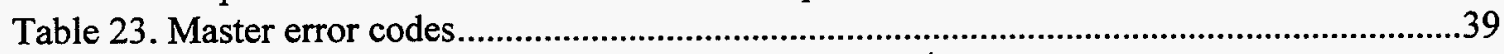

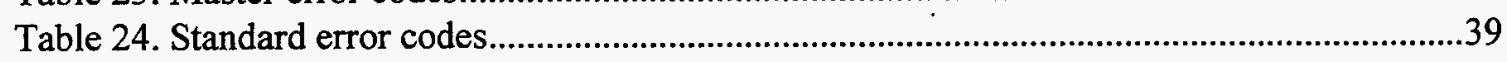

Table 25. Sensor module identification codes .........................................................................40 


\section{ACRONYMS}

$\mathrm{ac}$

CAP-WT capacitive weight sensor

CAVIS

dc

DIN

EPROM

EEPROM

ETX

GraFIC

IDC

$\mathrm{mA}$

MSV

NEMA

PCDU

PLD

RAD-SIP

RAM

ROM

SNM

SPB

SPCS

STX

TCP/IP

direct current

end of text character

milliampere

modular storage vault

random access memory

read only memory

special nuclear material

serial peripheral bus

start of text character alternating current

Continuous Automated Vault Inventory System

Deutsche Industrinorm, the standards setting organization for Germany

erasable programmable read only memory

electrically erasable programmable read only memory

Graphical Facility Information Center

insulation displacement connector

National Electrical Manufacturers Association

power and communications distribution unit

programmable logic device

radiation silicon pin diode

sensor polling and configuration system

Transmission Control Protocol/Internet Protocol 


\begin{abstract}
The purpose of this document is to describe the use and operation of the sensor concentrator in the Continuous Automated Vault Inventory System (CAVIS). The CAVIS electronically verifies the presence of items of stored special nuclear material (SNM). U.S. Department of Energy orders require that stored SNM be inventoried periodically to provide assurance that the material is secure. Currently this inventory is a highly manual activity, requiring personnel to enter the storage vaults. Using a CAVIS allows the frequency of physical inventories to be significantly reduced, resulting in substantial cost savings, increased security, and improved safety. The electronic inventory of stored SNM requires two different types of sensors for each item. The two sensors measure different parameters of the item, usually weight and gamma rays. A CAVIS is constructed using four basic system components: sensors, sensor concentrators, a data collection unit, and a database/user interface unit. One sensor concentrator supports the inventory of up to 20 items ( 40 sensors) and continuously takes readings from the item sensors. On request the sensor concentrator outputs the most recent sensor readings to the data collection unit. The information transfer takes place over a RS485 communications link. The data collection unit supports from 1 to 120 sensor concentrators ( 1 to 2400 items) and is referred to as the Sensor Polling and Configuration System (SPCS). ${ }^{1}$ The SPCS is connected by a secure Transmission Control Protocol/Internet Protocol (TCP/IP) network to the database/user interface unit, which is referred to as the Graphical Facility Information Center (GraFIC). ${ }^{1} \mathrm{~A}$ CAVIS containing more than 2400 items is supported by connecting additional SPCS units to the GraFIC. ${ }^{1}$
\end{abstract}

1. J. J. Dunigan et al., Graphical Facility Information Center Functional System Design Document, Y/DW-1636, Lockheed Martin Energy Systems, Inc., Oak Ridge Y-12 Plant, September 1996. 


\section{INTRODUCTION}

The sensor concentrator is a custom-designed electronic unit that concentrates the inventory sensor information from 20 items of stored special nuclear material (SNM) onto a sensor communications network. Fig. 1 shows a view of the bottom of the unit. The unit continuously monitors the stored material and, upon request, relays the sensor information to a data collection unit called the Sensor Polling and Configuration System (SPCS). ${ }^{1}$ Two sensors

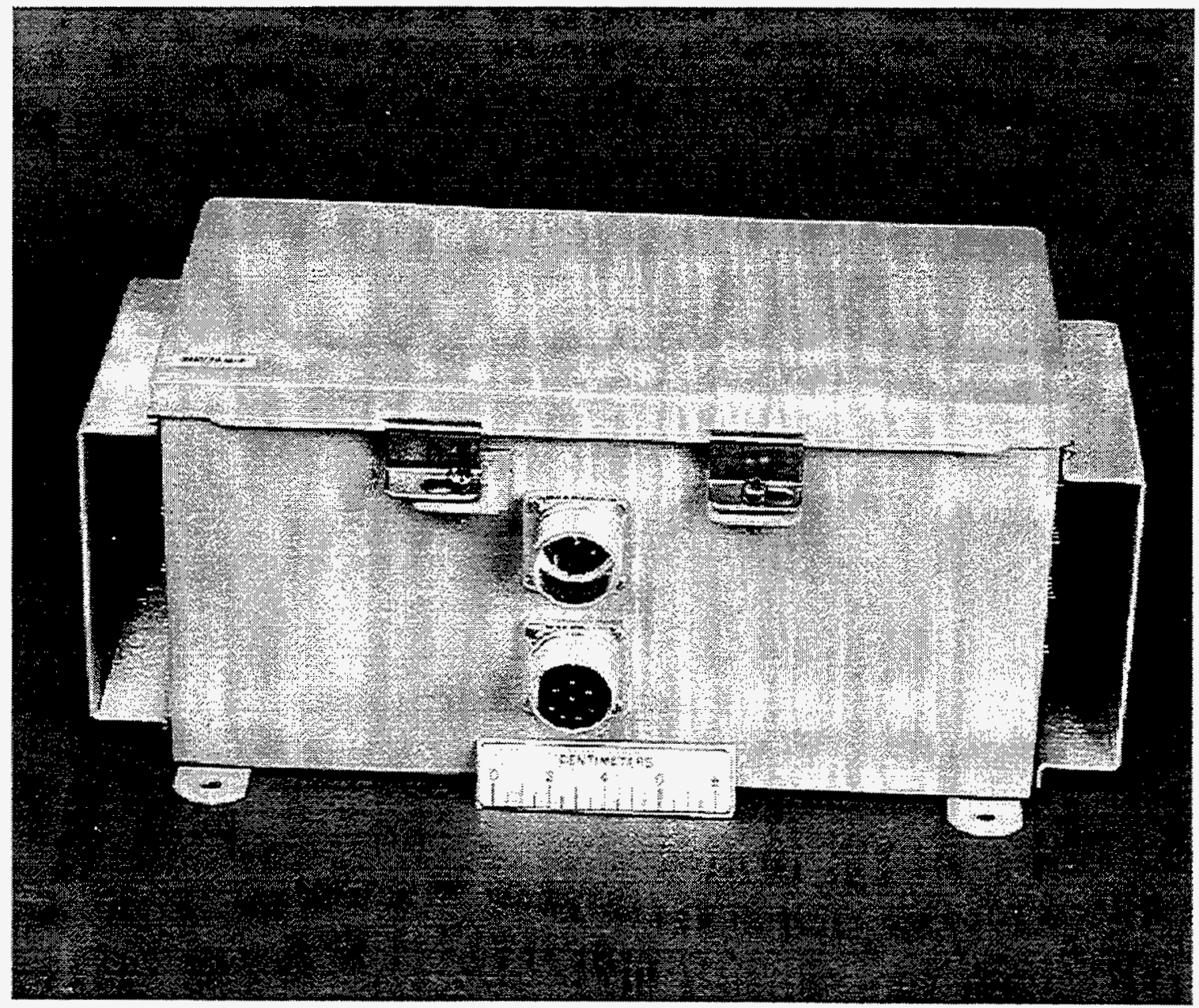

Fig. 1. Bottom view of the sensor concentrator.

that measure different parameters, usually weight and gamma rays, are required to verify the presence of a stored SNM item. The sensor concentrator groups sensor types as "Position- $A$ " or "Position-B" and can accommodate 20 of each type. This allows up to 20 items, each with a "Position- $A$ " and a "Position- $B$ " sensor, to be monitored by a single sensor concentrator. The sensor concentrator is housed in a $6 \times 6 \times 12$ in. NEMA enclosure that is mounted near the 20 items to be inventoried. When used with modular storage vaults (MSVs), ${ }^{1}$ the sensor concentrator is mounted directly onto the vault. To monitor more than 20 items of SNM, individual sensor concentrators are cascaded together. One SPCS can support up to 120 sensor concentrators, implementing a system capable of inventorying 2400 items of SNM. In a 2400- 
item system, information from each sensor is updated approximately once each minute. The sensor concentrator and the SPCS measure and store data readings only and do not establish alarm set points. Alarm set points are established in the Graphical Facility Information Center $(\text { GraFIC })^{1}$, which tests for alarm conditions.

Sensor concentrators connect to a communications network and power source through daisy chain cables. A male and a female 8-pin circular connector are located on the bottom of the unit for this purpose(Fig. 1). These connectors connect adjacent units together and pass the power and communication sources through to the next sensor concentrator. Groups of sensor concentrators daisy-chained together connect to a power and communications distribution unit (PCDU). One PCDU supports up to 15 sensor concentrators. To implement an inventory system with 120 sensor concentrators, 8 PCDUs are connected to a single SPCS. The SPCS connects to the GraFIC via a private TCP/IC communications network. The complete system containing all the sensors, sensor concentrators, SPCS, and GraFIC is referred to as a Continuous Automated Vault Inventory System (CAVIS). This document focuses on the sensor concentrator and how it relates to the sensors and the SPCS. 


\section{SENSOR CONCENTRATOR DESCRIPTION}

The sensor concentrator electronics is a modular design using circuit cards that plug into a custom, six position motherboard. Three motherboard connectors are visible in Fig. 2 , in which modules have been removed. The plug-in modules use 96-pin male Deutsche

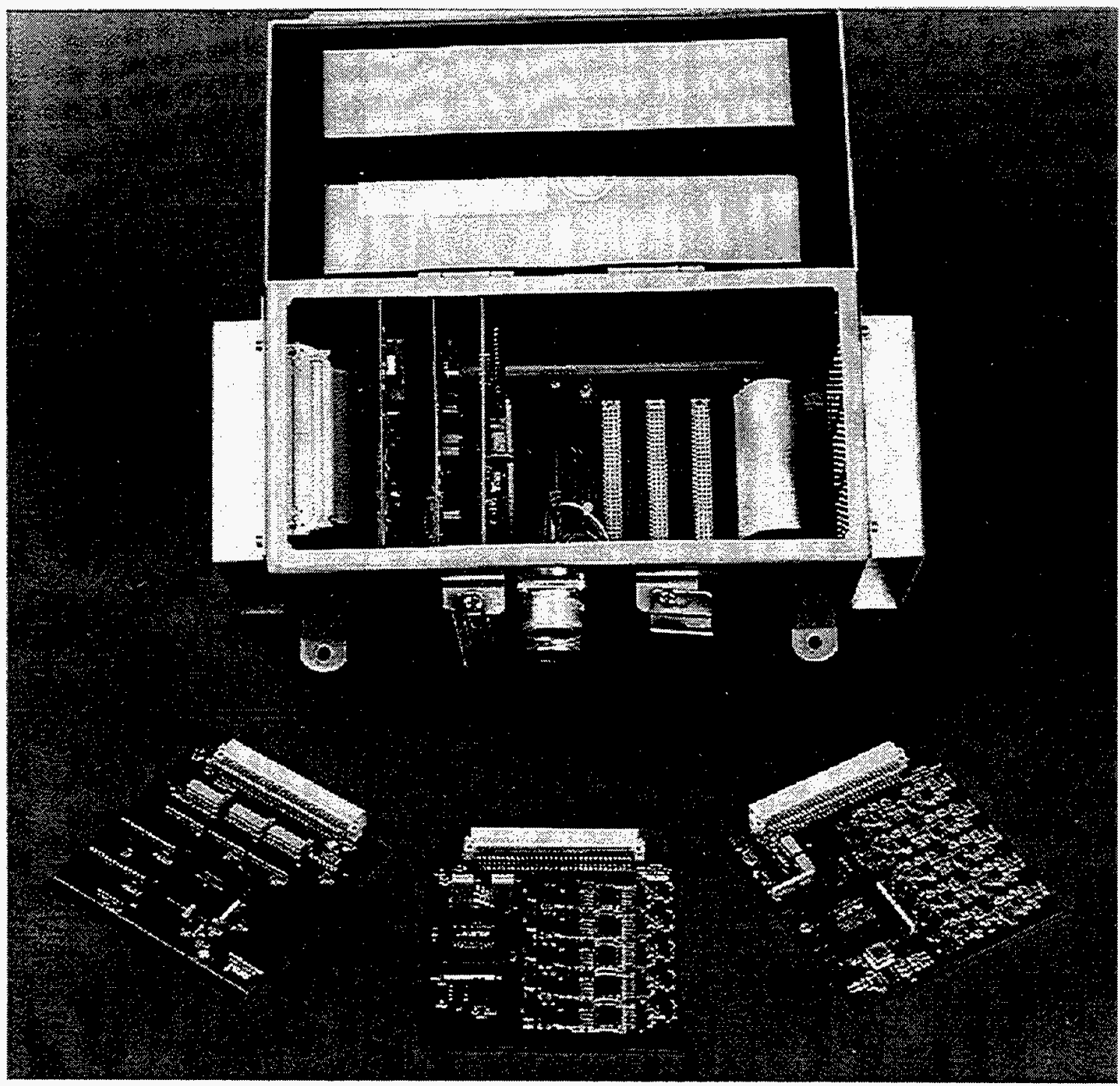

Fig. 2. Inside view of the sensor concentrator with three module removed.

Industrinorm (DIN) connectors and mate to female DIN connectors on the motherboard. The sensor concentrator distributes the monitoring of $20 \mathrm{SNM}$ items between two electrical sections. Each section monitors ten "Position- $A$ " sensors and ten "Position- $B$ " sensors and contains an independent processor module, "Position- $A$ " sensor module and "Position- $B$ " sensor module. The "Position- $A$ " and "Position- $B$ " naming convention is used to tag the location of sensor data and does not refer to a specific type of sensor. Monitoring of the two sensors attached to a stored item of SNM is divided between the separate sections. One sensor is connected to a "Position- 
A" sensor module in one section, and the other sensor is connected to a "Position-B" sensor module in the other section. This design provides some redundancy at a reduced monitoring level. Even if one complete section of the electronics fails, measurements from one sensor on each SNM item is continued by the other section. In a case where only one section is operating, ten of the items are monitored by "Position- $A$ " sensors and ten by "Position-B" sensors.

The two electrical sections are integrated in the same enclosure and appear physically as one unit. Figure 2 shows the sensor concentrator with the processor module and both sensor modules removed from the right side. The sensor module positions located at the far left and right of the motherboard report data as "Position-A" sensors. The sensor module positions located between the processor modules and the "Position- $A$ " modules report data as "Position-B" sensors. The sensor data reporting is described in Sect. 2.6. The four sensor positions in the motherboard are generic and support any sensor module correctly designed. In fact, all four sensor modules can be the same type. This approach allows different sensor types to be supported by a single sensor concentrator design. If future requirements dictate different sensor types or that existing sensors be updated, new sensor modules can be designed to be used with the same concentrator. This assumes that any new sensor meets the interface requirements of the sensor concentrator(Sect. 5). At the time of this writing, only two sensor modules have been designed, one for a capacitive weight sensor and the other for a counts per second gamma ray detector. Descriptions of these modules are given in this Sect. 2.4 and 2.5.

\subsection{SENSOR CONCENTRATOR FUNCTIONAL BLOCKS}

As previously stated, the sensor concentrator electronics is divided into two sections. Electrically, each group of ten SNM items has ten "Position-A" sensors monitored by one section and ten "Position-B" sensors monitored by the other section. Physically, all 20 sensors in one group are connected to the left sensor input panel and all 20 sensors in the other group are connected to the right panel. The sensor input panels are represented by the extreme left and right blocks in the functional block diagram of Fig. 3. The left and right half of the diagram appear to contain separate, independent electronic sections. A closer inspection reveals that this is not the case. Note that the "Serial Peripheral Bus 1" and the "Serial Peripheral Bus 2" cross between the two sections. These buses are used to communicate and transfer data between the processor module and sensor interface modules. Each "Position-B" sensor interface module is connected to the serial peripheral bus of the opposite side. In this manner, the "Position- $\mathrm{B}$ " sensors physically connected to one side are monitored by the processor module on the other side. The serial peripheral buses are three wire buses designed to Motorola specifications. ${ }^{2}$ Using these buses significantly reduces the number of connections required in the motherboard.

The blocks in Fig. 3 titled "Network In" and "Network Out" represent the two circular 8pin connectors referred to in Sect. 1. As stated, these connections serve to daisy-chain sensor concentrators together and provide both power and communication connections to the unit. The power and communication wiring is routed between the connectors passing the connections through to the next unit; however, a single unit can be operated without any connection to additional units. 


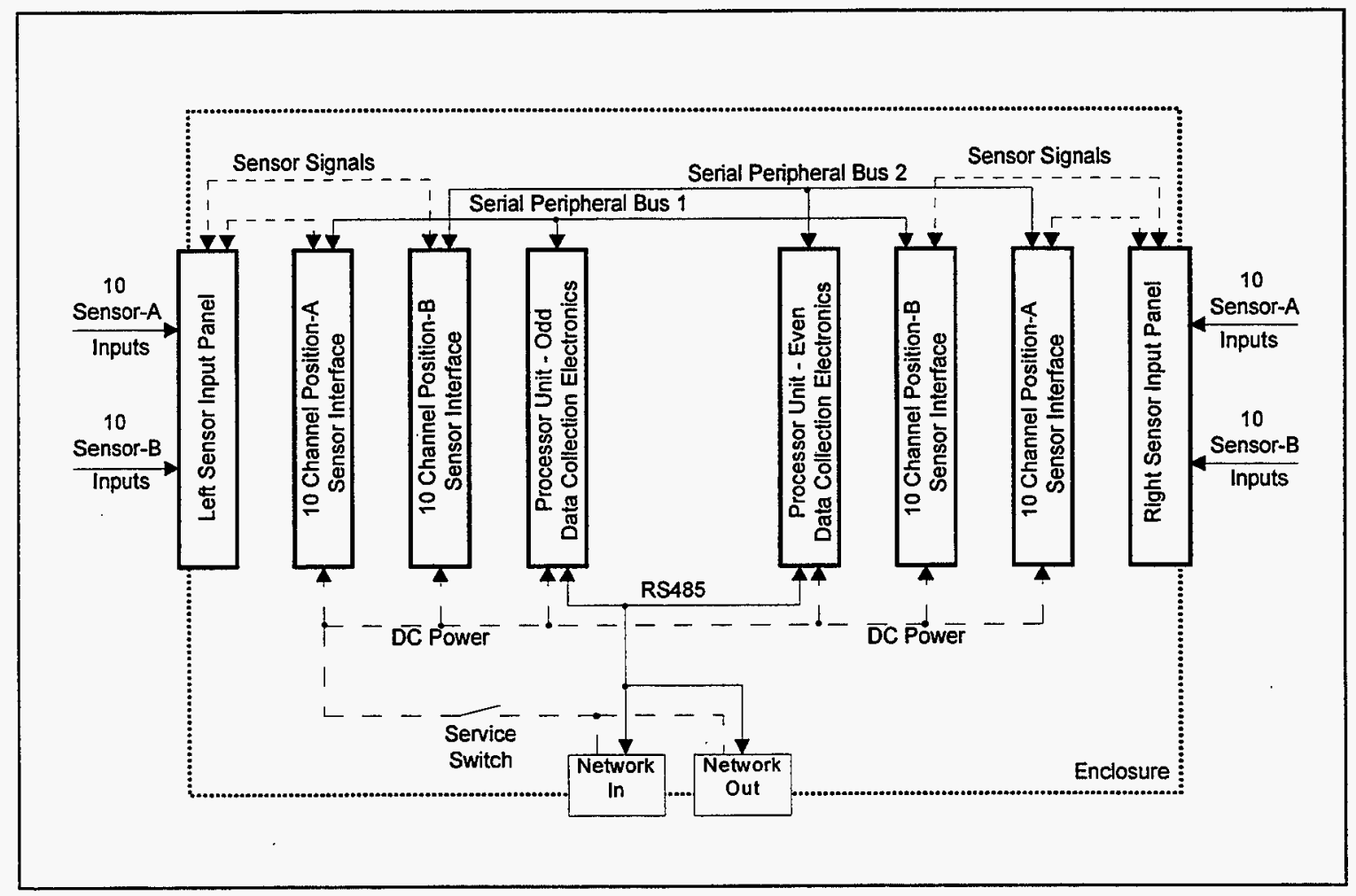

Fig. 3 Sensor concentrator functional block diagram.

The SPCS and sensor concentrators communicate using a full duplex RS485 bus in conjunction with custom software. The concentrators are designed to operate with a dc input voltage between 11 and 14 . The power and communication connections are distributed from the connectors to the motherboard by a wiring harness. This wiring harness is visible in Fig. 2 . The power and communication connections are represented in Fig. 3 by the dashed and solid lines in the lower portion of the diagram. Up to 15 concentrators are powered by a single power supply in the PCDU. The PCDU is described in Sect. 4 of this document. Designing the sensor concentrator to operate down to $11 \mathrm{~V}$ compensates for any voltage drop that may occur in the cable system. The raw dc input voltage is distributed to each module position by the motherboard. Modules do not use the input power directly, and all designs are required to use voltage regulators or dc-to-dc converters. The service switch illustrated in Fig. 3 removes all power from the motherboard but does not affect other sensor concentrator units on the same cable system. Using this switch allows modules to be replaced in one unit without powering down all the units that share the same PCDU. The only shared components between the two separate electronic sections are the motherboard, power switch, input and output connectors, and wiring harness. Each processor module provides individual RS485 line drivers and receivers. Failure in any of these shared components will cause complete loss of the unit, but since these components do not contain active electronic devices, reliability is high. 
' I '8!

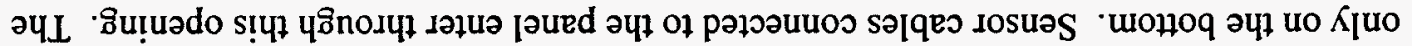

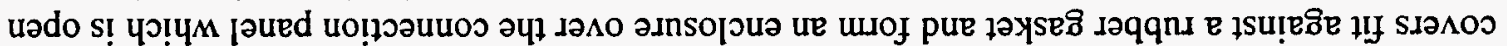

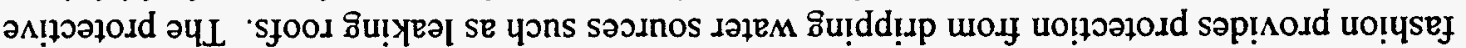

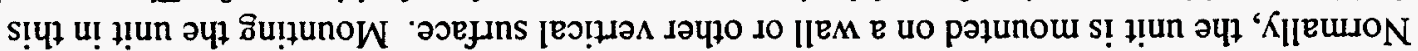

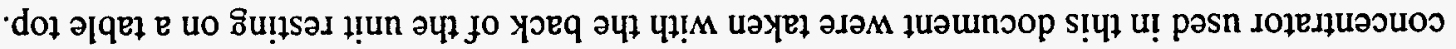

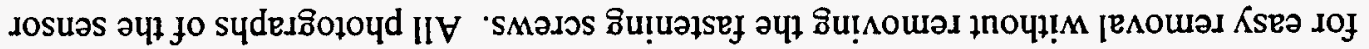

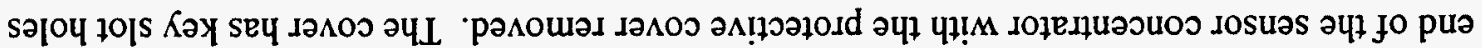

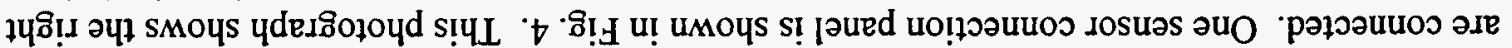

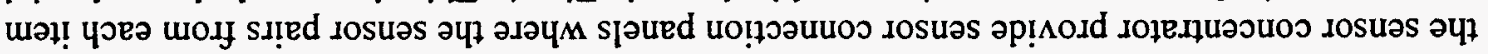

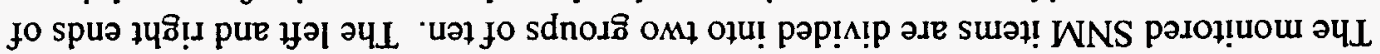

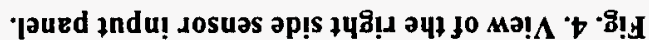

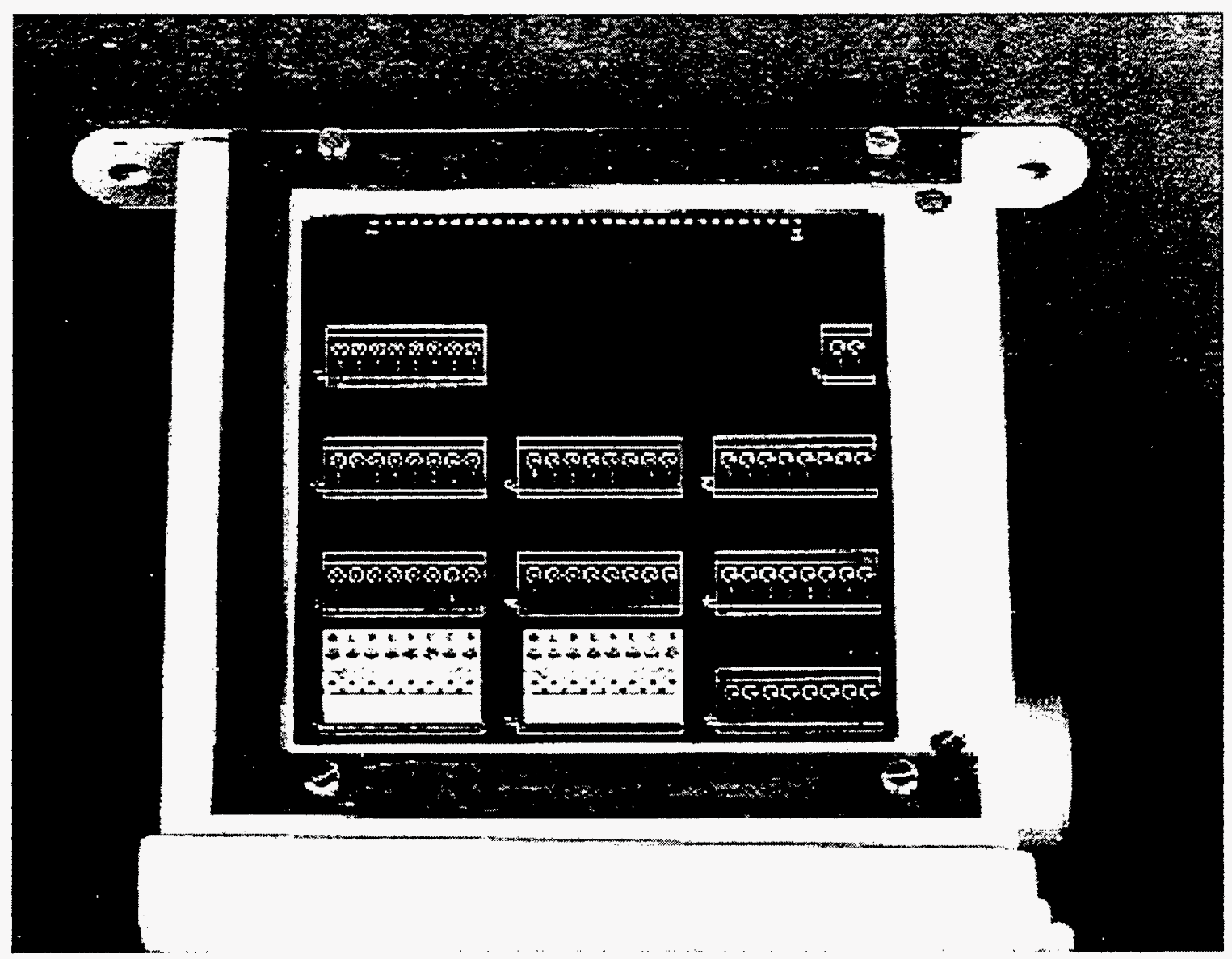




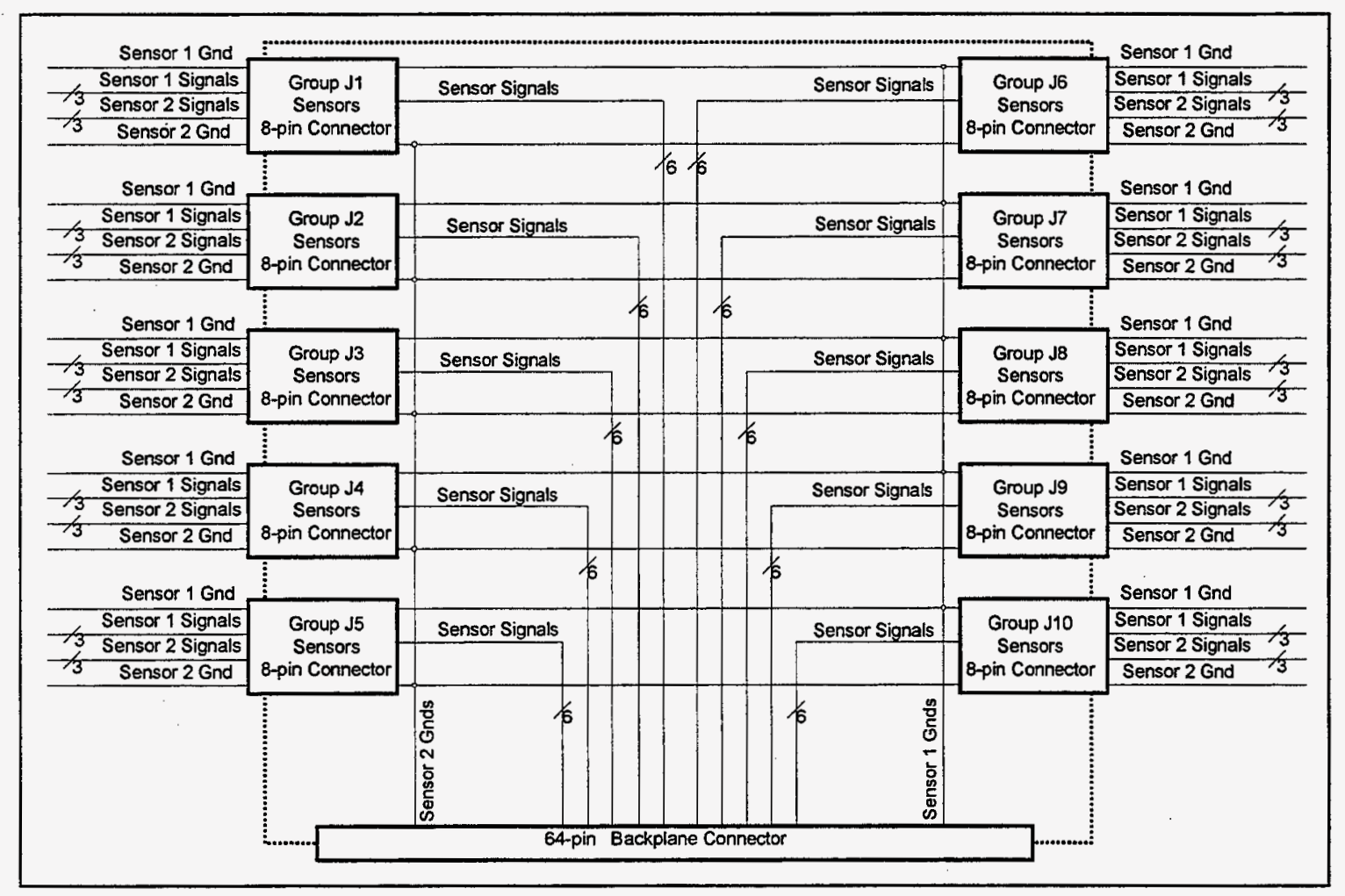

Fig. 5. Functional diagram of the sensor input panel.

Note the ten groups of eight pins shown in Fig. 4. Although the lettering is not clearly visible in the photograph, these groups of pins are labeled J1 through J10. Each group connects a pair of sensors from one of the ten SNM items. The single pair of pins in the lower left corner of the panel, labeled J11, are reserved for future use. Each group of eight pins furnishes the connections for one "Position-A" and one "Position-B" sensor. Four of the pins are assigned to each sensor and provide the necessary power and signal connections. Three pins are dedicated signal paths, and one pin is ground. Figure 5 is a functional diagram of the sensor panel. All the grounds shown in Fig. 5 are common, but each signal path is unique. The signal paths are a direct connection to the sensor interface module and can be used to supply power or input and output signals to the sensor. A 64-pin insulation displacement connector (IDC) is used to make all the connections to the motherboard. Each sensor connected to the panel is assigned 3 dedicated signal lines for a total of 60 . Two pins on the connector are used for ground connections and the remaining two connect $\mathrm{J} 11$ to the motherboard. A short ribbon cable connects the panel to a mating 64-pin connector on the motherboard. The ribbon cables connecting the left and right panels to the motherboard are visible in Fig. 2. To make sensor connections to the panel, either a single 8-position terminal block connector or two 4-position terminal block connectors are pushed onto the desired group of pins. For illustration purposes, two 8 position connectors are pushed onto $\mathrm{J} 1$ and $\mathrm{J} 5$ on the panel shown in Fig. 4. The push-on terminal block connectors are normally attached to the sensor cables and pushed onto the panel when the sensors are installed. Optionally, empty connectors can be installed onto the panel and the sensor cables cut and dressed in the field. The second method is advantageous in installations where the distances between the items and the sensor concentrator are not predetermined. 


\subsection{PROCESSOR MODULE DESCRIPTION}

The processor module determines the configuration of the electrical half of the sensor concentrator it occupies. In Fig. 2 the processor module is the left circuit board displayed in front of the sensor concentrator. Only hardware functions common to all sensor modules are located in this module. The main purpose of the module is to collect data from the two sensor interface modules in the same section and report that data when polled. The sensor data in the module's memory is updated at a rapid rate but is reported only at the polled rate. The SPCS sets the poll rate for the system. The processor module accepts commands form the SPCS and, depending on the command, sends requested data, performs diagnostics, or performs setup and installation procedures. The firmware located in the processor module memory is designed to interrogate the two sensor interface module slots and determine the sensor module type inserted. This initialization process is performed each time power is applied to the unit. Sensor concentrators can be operated with empty sensor module positions if necessary. The firmware contains data acquisition routines for all known sensor boards. The initialization process selects the correct routine for each sensor module position based on the module type detected. The firmware will report an error if the SPCS requests data from a slot that does not have a sensor module installed.

Data packets reported back to the SPCS identify the sensor module type, the module location, and the address of the processor module originating the packet. A detailed description of the firmware and communications protocol is given in Sect. 6. Module locations are determined by connections on the motherboard at the time the module is inserted. The left side of the sensor concentrator is identified as the odd side and the right as the even side. A processor module becomes either odd or even based on its location. Each module is uniquely identified by a 48-bit serial identification (ID) number embedded in the internal electrically erasable programmable read only memory (EEPROM) of the microprocessor. The node address for the unit is also stored in this memory. When the sensor concentrator is connected to an authorized installation device, the serial ID and the node address can be programmed. The serial ID can be programmed only once. If programmed incorrectly, the module must be removed and special steps taken to correct the error. All node addresses programmed into processor modules are even. In a multiple sensor concentrator system, all odd addresses are skipped. Normally, both processor modules installed into the same unit will be programmed with the same even node address. The position a processor module is inserted in, odd or even, determines whether the module responds to the even address or the even address plus one. For example, if the modules are programmed with the address 20 , the module inserted in the left side (odd) will respond to the address 21 .

A block diagram of the processor module is illustrated in Fig. 6. The heart of the module is the Motorola MC68HC11F1 microprocessor. The F1 version supports a full $64 \mathrm{~K}$ of external address space, half of which is consumed by random access memory (RAM) and half by erasable programmable read only memory (EPROM). The module firmware is stored in the EPROM memory and the RAM provides storage for temporary data and variables. The reset circuit initializes the microprocessor each time the module is powered on. The manual reset switch is provided for maintenance use. Eight digital output and eight digital input lines are provide for module control and handshaking. These lines are labeled $\mathrm{m}$-out and $\mathrm{m}$-in in the diagram. Integrated circuits provide buffering for these lines, the serial peripheral bus (SPB) and the E-clock (E clk). 


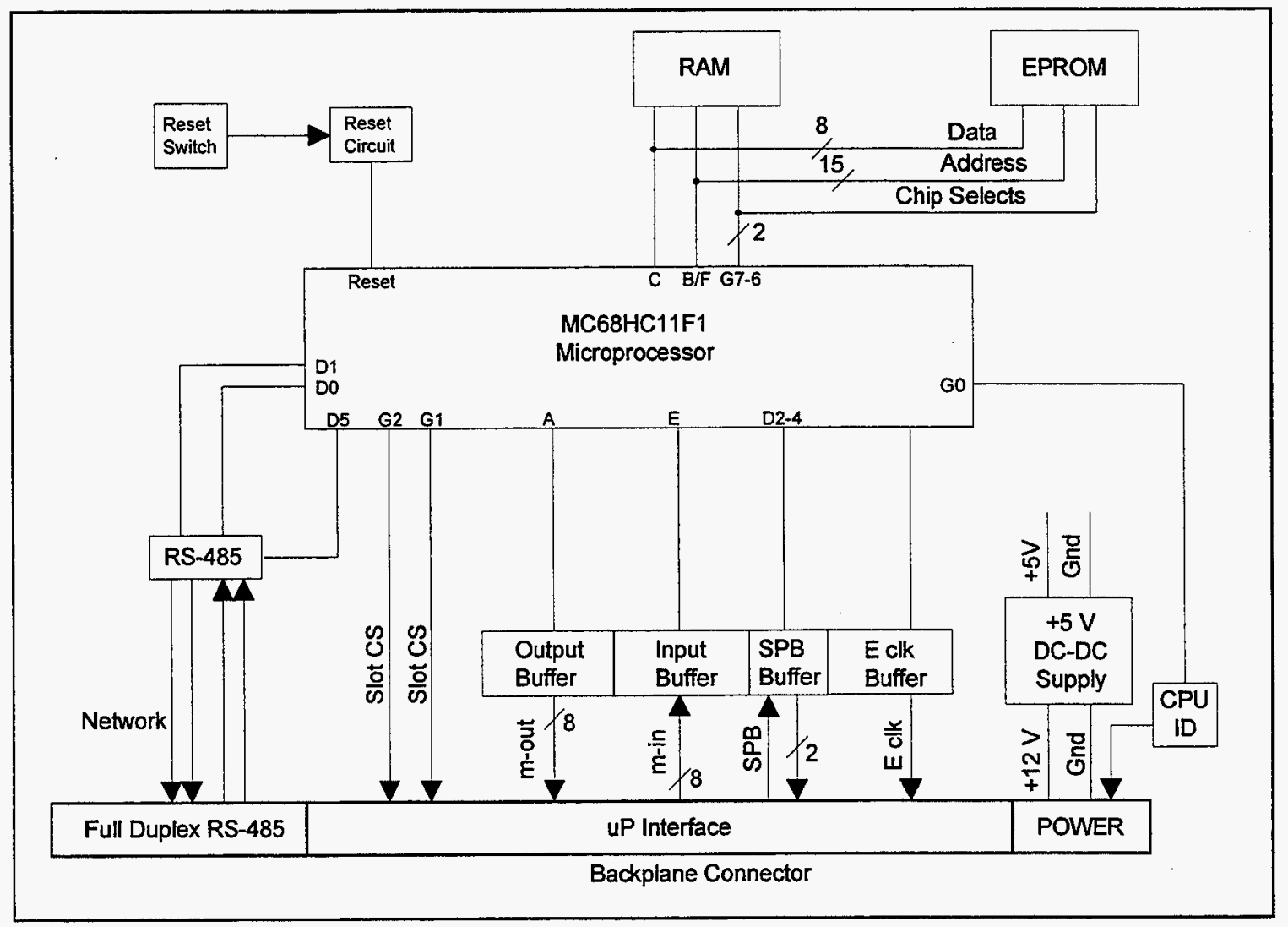

Fig. 6. Processor module block diagram.

As mentioned in Sect. 2.1, the SPB is used to transfer data between the processor and sensor modules. Two slot chip selects (Slot CS) provide signals to enable the individual sensor modules. The m-out, $\mathrm{m}$-in, and SPB lines run to both modules. Only the module with the Slot $\mathrm{CS}$ enabled will make active connection to the buses. The processor module ID senses whether the processor module should be odd or even by detecting the position logic state on the motherboard. The POWER and Full Duplex RS-485 signals originate directly from the daisychain connectors via the motherboard. These signals are common to both processors. The only power required by the processor module is $+5 \mathrm{~V}$. A single voltage regulator drops the $+12-\mathrm{V}$ supply down to provide the $+5 \mathrm{~V}$. The $\mathrm{MC} 68 \mathrm{HC} 11$ contains a standard hardware serial communications interface with a nonbuffered, single-ended output and input. This interface is buffered and conditioned to the RS-485 standard by a special integrated circuit designed for that purpose.

\subsection{GAMMA SENSOR INTERFACE DESCRIPTION}

Currently only one type of gamma sensor interface module is designed for the sensor concentrator. The center circuit board displayed in the foreground of Fig. 2 is the gamma sensor module. This module supports a custom, low-cost gamma detector referred to as RAD-SIP derived from "RADiation Silicon Pin diode". ${ }^{3}$ Up to ten of these detectors are connected to a single module. The main elements of the detector are a silicon photo detector, a low-noise preamplifier, and a pulse-shaping amplifier. The silicon is sensitive to gamma energies up to about $90 \mathrm{keV}$. Energies above that level are inferred from the Compton effect. Circuits on the 
sensor interface module discriminate the pulses generated by the detector and ignore gamma energies below $60 \mathrm{keV}$. The higher energy pulses are converted to digital logic pulses and are counted by circuits on the module. The counts for all ten sensors are accumulated simultaneously during a 1 second interval. At the end of this interval the ten accumulated counts are retrieved and stored by the processor module. The processor module integrates the counts

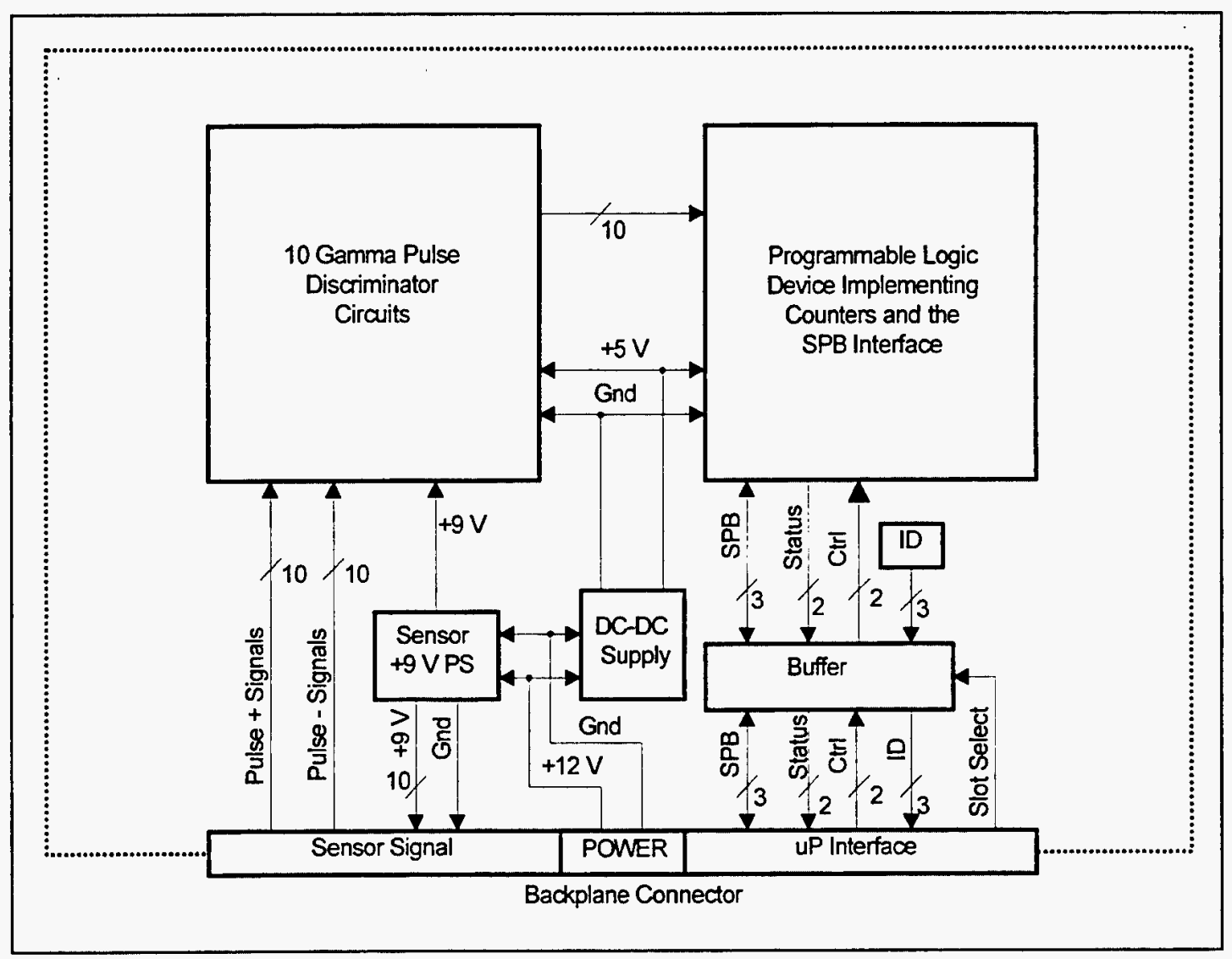

Fig. 7. Functional diagram of RAD-SIP sensor module.

over a longer period of time, updating the readings each second.

A functional block diagram of the gamma sensor module is illustrated in Fig. 7. The large block in the left half of the diagram represents the module circuits that perform pulse discrimination and conversion. Each sensor is provided with an independent discriminator and pulse conversion circuit. An adjustment in each circuit is set to calibrate the pulse discriminator. Three dedicated connections are made from the module to each sensor. These connections are pulse positive signal, pulse negative signal, and $+9 \mathrm{~V} \mathrm{dc}$. All ten sensors attached to the module share a common ground. If a single $+9-\mathrm{V}$ regulator were used to power the ten gamma circuits and detectors, the failure of one detector could short out the entire $+9-\mathrm{V}$ supply. This would cause all ten gamma detectors to cease operation even though only one failed. To guard against this possibility, five individual $+9-\mathrm{V}$ regulators are used. Each regulator powers two gamma detectors and the associated circuits. This design allows the other eight detectors to continue operation in the event that one detector shorts out the $+9-\mathrm{V}$ supply. One single $+5-\mathrm{V}$ regulator provides all the other power requirements of the module.

The large block on the right side of the diagram represents a 4000 -gate programmable logic device (PLD). The digital logic for ten accumulating counters and a SPB interface is 
implemented with this integrated circuit. The digital output signals from the gamma circuits connect to the counters. Two digital control lines (ctrl) from the backplane are buffered and fed to the PLD. These control lines are used by the processor module to start, stop, and reset the accumulation counters. The actual timing for the accumulation window is determined by timing circuits in the processor module. Three logic states are buffered and sent to the backplane for module ID. These logic states enable the processor module to determine the sensor module type during initialization. The processor module controls the slot select to connect the sensor module to the interface bus. The two sensor modules controlled by each processor have individual slot selects. These slot selects affect only the connection of the module to the backplane and communications with the processor module. The other sensor-related functions of sensor modules operate continuously. Only one sensor module in each section is enabled at the same time by the processor module; this is necessary to avoid contention on the backplane.

The logic circuits and counters contained within the PLD are designed using a workstation software tool. This tool outputs the finished design as a binary image. Loading the image into the PLD device causes the device to emulate the logic of the design. The terms volatile and nonvolatile can be used to classify the many programmable logic devices available. Most PLD manufacturers produce both device types. In the volatile device, the binary image is stored in temporary memory and must be reloaded each time the circuit power is interrupted. The nonvolatile device uses a permanent-type memory and retains the design when powered off. Each device type has distinct advantages that must be compared when selecting the PLD type for a particular application. The sensor modules designed for the sensor concentrator use a volatile device. These PLD devices consume less power than their nonvolatile equivalents and are an excellent choice for low-power applications. Reduced power consumption was the deciding factor in the decision to use the volatile device for sensor concentrator designs. Reducing the current used by individual concentrators reduces the overall power requirements of the monitoring systems and minimizes voltage drop in distribution wiring.

The gamma sensor module provides two methods to load the binary image into the PLD. One method employs a serial, 8-pin, one-time programmable read only memory (ROM). This ROM is programmed with the binary image and is inserted into a socket provided expressly for that purpose. Jumpers select which method of PLD loading to use. If the jumpers are set to select the ROM option, the device is automatically loaded from the ROM each time power is applied. The second method loads the PLD with the processor module via the SPB. In this case the binary image is programmed with the firmware and stored in the processor module. The two buffered status lines in Fig. 7 are read by the processor module to detect if the image loaded successfully.

\subsection{WEIGHT PAD SENSOR INTERFACE DESCRIPTION}

Of the two types of sensor interface modules currently designed, one is the RAD-SIP interface and the other a capacitive weight sensor interface. This sensor is sometimes referred to as CAP-WT derived from CAPacitive Weigh and Temperature. The CAP-WT contains circuits that measure the change in capacitance between plates and the ambient temperature. To improve accuracy and allow operation in varying environments, the capacitance circuit is compensated for humidity changes. The CAVIS is designed to support sensors with two parameters. Dual parameter sensors are treated as a single unit. The CAP-WT sensor is a dual parameter sensor. Up to ten CAP-WT sensors are connected to a single interface module. Both the weight and temperature of a stored item of SNM are conveyed back to the sensor interface module in a single periodic digital signal. The pulse portion of the signal represents weight, and the period 
portion represents temperature. A unique feature of the CAP-WT sensor is that the measurement information and the power share the same two conductor cable. In effect, the sensor modulates the power supply to transmit information back on the power conductors. One of the main functions of the sensor interface module is to demodulate this information from the power and translate it into $\mathrm{a}+5-\mathrm{V}$ logic pulse. The sensor module contains the necessary circuits to demodulate and measure the pulse and period of ten capacitive weight sensors signals.

The RAD-SIP and CAP-WT interface modules are similar in several ways. This is evident when the functional diagram for the CAP-WT module, Fig. 8, is compared with Fig. 7. The information contained in Sect. 2.4 regarding the use and loading of programmable logic also applies to this module. The processor module communicates with the PLD using an interface identical to the one used on the RAD-SIP. The same two options for loading the PLD are available on the capacitive weight interface module. The logic implemented with the PLD on this module, with the exception of the processor interface, is completely different. Additionally, a $10-\mathrm{MHz}$ time base is provided for pulse and period measurements. The PLD contains a pair of counters, rather than ten, that are connected to the individual weight sensor signals through a multiplex circuit. One counter is used to measure the period of the signal and the other to measure the pulse width. The counters operate in a one-shot mode and are gated on and off by

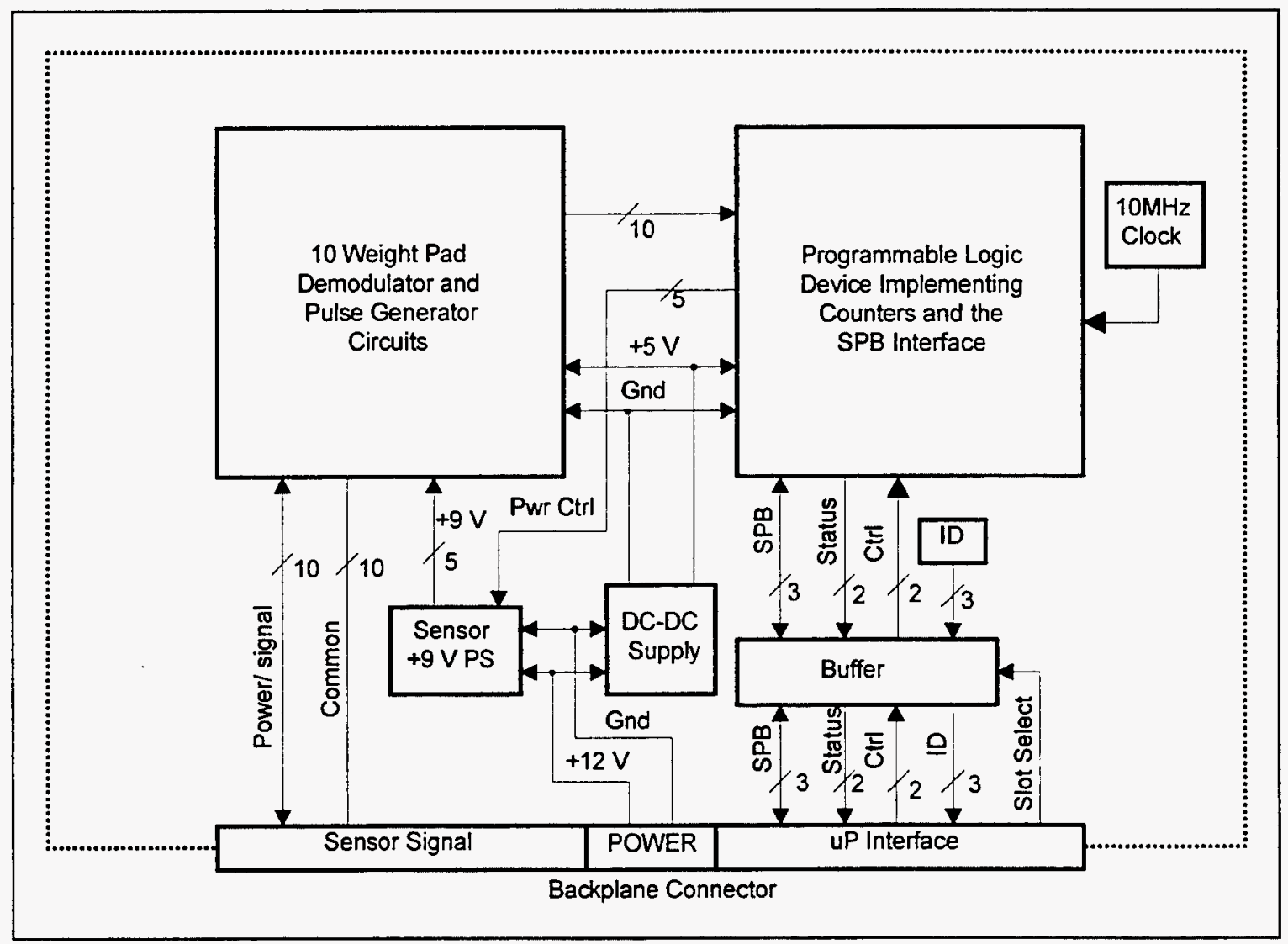

Fig. 8. Functional diagram of CAP-WT sensor module.

the edges of the sensor signal.

To control the measurement sequence, the processor module sends commands and retrieves data via the SPB. To begin a measurement, the processor first powers up a pair of weight sensors. Weight sensors are powered on in pairs because a single circuit controls both. 
After waiting for the power to stabilize, an individual weight sensor is selected. Next the counters are signaled to arm through the control lines (ctrl). Once armed, the period and pulse counters wait for the next negative edge of the sensor signal and then start counting $10-\mathrm{MHz}$ clock periods. The pulse counter stops when the positive edge of the signal is detected. The period counter waits for the next negative edge before stopping. After both counters have finished, a done flag is set. The processor module checks the done flag through the SPB and reads the counters after the flag becomes true. This sequence is repeated selecting a different weight sensor channel each time. The firmware integrates and filters the pulse and period values and updates the stored readings every 1.6 seconds.

The module identification and $+5-\mathrm{V}$ power requirements are provided in the same fashion as they are on the RAD-SIP module. The two modules differ in the design of the $+9-\mathrm{V}$ power supply. Both modules have five voltage regulators, each powering two sensors, but unlike the RAD-SIP module, the CAP-WT regulators are turned on and off by control lines. Only one regulator is turned on at a time. Cycling the voltage regulators reduces the power consumption of the sensor concentrator. A significant power reduction is obtained by using this method. Only 4 out of the 20 weight sensors connected to a sensor concentrator will be powered on at any given time. Each individual regulator powers a specific pair of sensors. One regulator powers sensors 1 and 6 , the next 2 and 7, then 3 and 8,4 and 9, and finally 5 and 10 . The number of times regulators must be cycled is cut in half by reading both sensors in the same cycle. The firmware reads the sensors in the order $1,6,2,7,3,8,4,9,5,10$. This operating sequence was chosen to guard against cross talk between adjacent signal traces on circuit boards.

There are no adjustments on the capacitive weight sensor interface module. All calibration adjustments are made in the sensor at the time of manufacture. Note in Fig. 8 that only 20 connections are made from the module to the sensors. The large block in the left half of the diagram contains the circuits that demodulate the sensor signals. After being converted to a $+5 \mathrm{~V}$ digital level, these signals are input to the PLD for measurement.

\subsection{RELATIONSHIP BETWEEN SENSOR LOCATION AND DATA}

The SPCS must send four commands to the sensor concentrator to collect data from all 40 sensors. Each processor module stores the data from one "Position- $A$ " and one "Position- $B$ " sensor module. Slots 1 and 4 of the sensor concentrator report "Position- $A$ " data, and slots 2 and 3 report "Position-B" data. The relationship between position data and slot location is shown in Fig. 9. The processor on the left side of the concentrator responds to odd addresses and the one on the right to even addresses. The odd processor retrieves data from sensor modules located in slots 1 and 3 (odd numbers) and the even processor from slots 2 and 4 . The SPCS sends a "Report-A" command to the odd processor to collect data from sensors monitored by the module in slot 1. A "Report-B" command is sent to the even processor to collect data from the sensors monitored by slot 2 . Sending "Report-B" commands to the odd processor returns data from slot 3 , and sending "Report- $A$ " commands to the even address returns data from slot 4 . The data are transmitted in packet form, and one report returns the data for 10 sensors. The data are ordered in the packet by the position in which the sensors are connected to the input panel. Refer to Sect. 2.2 for an explanation of the input panel. One report packet contains the data from 10 sensors. Four report commands in the sequence described previously will return the data from all 40 sensors. Each report packet contains the source node address and the sensor module type. Refer to Sect. 6 for a more detailed description of the packet contents. 


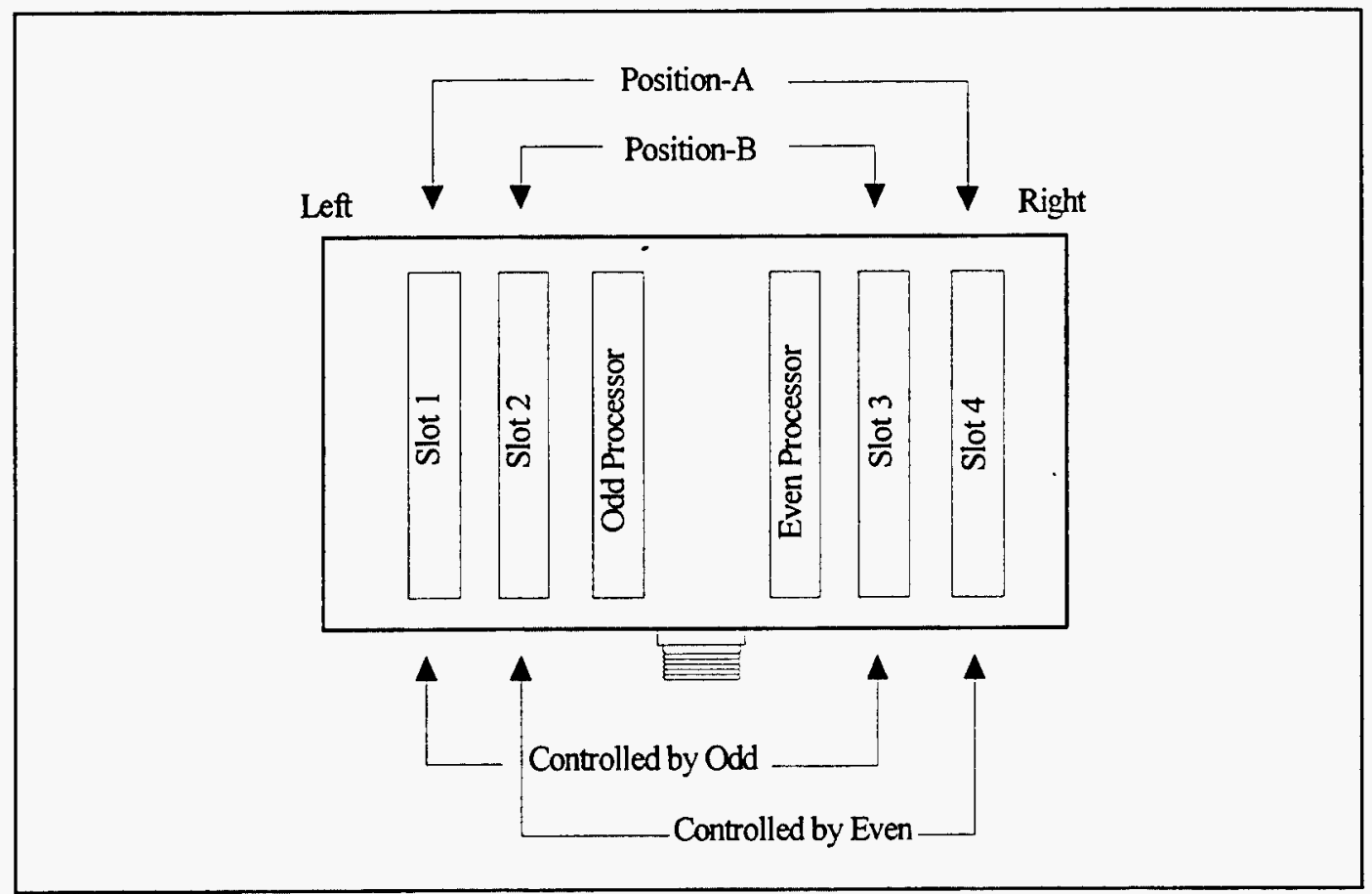

Fig. 9. Diagram of how data relate to slot position. 


\section{STANDALONE SENSOR CONCENTRATOR OPERATION}

The sensor concentrator can be operated as a single unit outside the context of the CAVIS; this is convenient for special monitoring requirements or demonstrations. As few as one sensor may be attached. When operated in this fashion custom software and a personal computer are required to substitute for the GraFIC system and the SPCS. In addition, an RS232 to RS485 converter and a 12-V power supply can be used to replace the PCDU. The converter translates the personal computer communications port to the full duplex RS485 network necessary to communicate with the sensor concentrator. A photograph of a sensor concentrator connected as a demonstration unit is shown in Fig. 10. The communications converter and the power supply are out of view to the left of the photograph. The white cable connects the computer communications port to the RS232 to RS485 converter. The black cable connects the sensor concentrator to the RS485 side of the converter and the power supply.

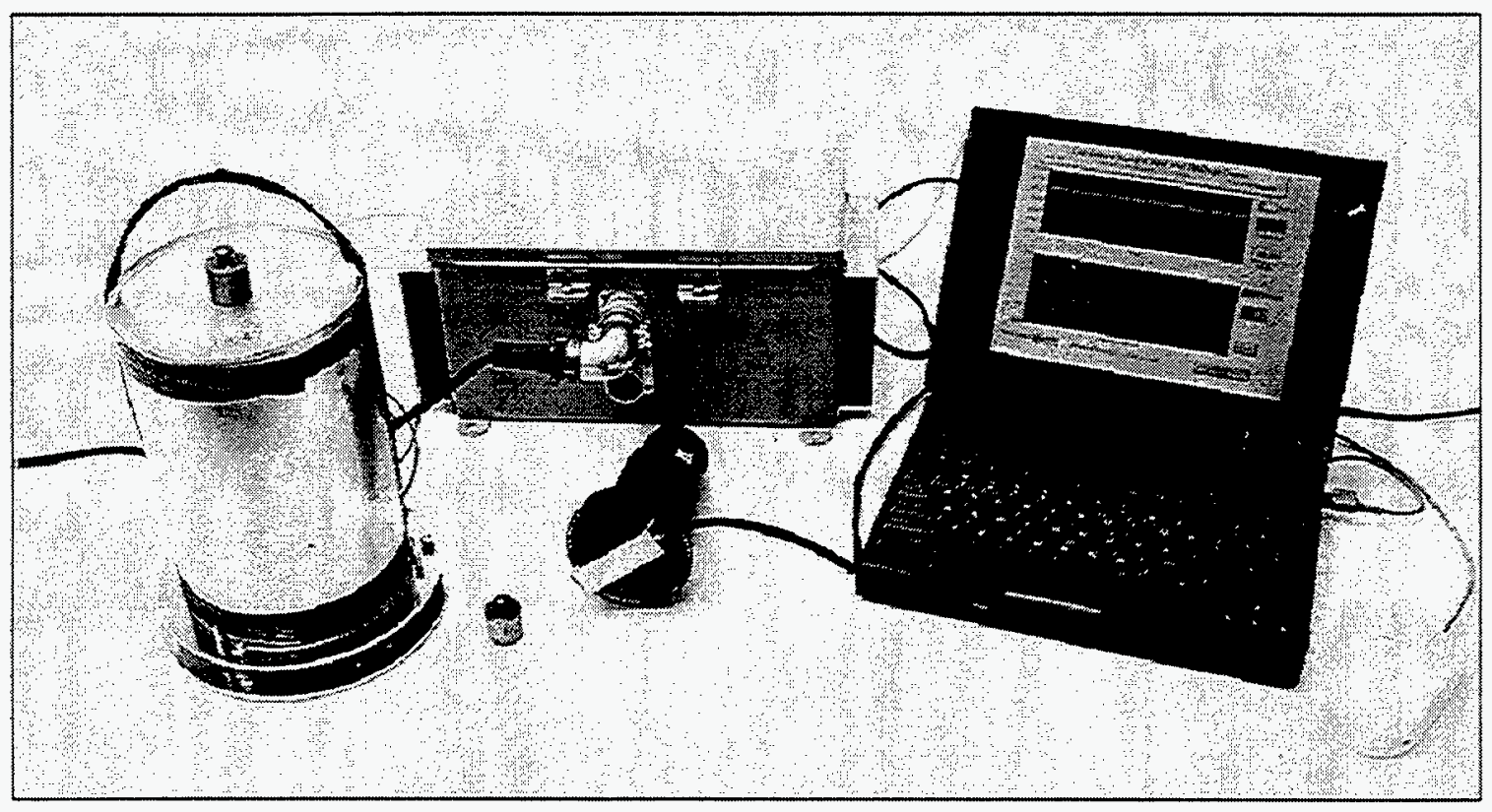

Fig. 10. Photograph of a CAVIS sensor demonstration.

The demonstration unit has one CAP-WT sensor and one RAD-SIP gamma sensor attached. The CAP-WT sensor is pictured on the left side. A simulated item of SNM is placed on the CAP-WT sensor. Two small brass weights are pictured, one on top of the simulated item and one resting on the table. These are added and removed during demonstrations to show weight change. The black item directly in front of the sensor concentrator is an old camera lens. The lens has a piece of velcro glued to the optical surface, allowing attachment of a RAD-SIP sensor. Normally the RAD-SIP sensor is mounted inside the CAP-WT sensor and measures gamma from the SNM item. Since the simulated SNM item contains only a steel weight that is not radioactive, the camera lens is used as a source. Older camera lenses were sometimes coated with thorium to increase the index of refraction. The thorium emits high-energy gamma rays that are easily detected by the RAD-SIP sensor.

The lap top computer in Fig. 10 is running a custom software program written specifically for this demonstration setup. The top portion of the screen graphically shows the 
last 10 seconds of the CAP-WT sensor output. The output is also provided in numerical format to the right of the graph. The bottom half of the screen displays the RAD-SIP sensor output in the same manner. The sensor concentrator has also been used in stand-alone configurations to take long-term data from actual items of SNM. A different custom software program designed to log data for long periods of time is used in these applications. 


\section{MULTIPLE SENSOR CONCENTRATOR OPERATION}

A single sensor concentrator is capable of monitoring 20 items of SNM. Systems for monitoring larger number of items are constructed by cascading individual sensor concentrators together. The units are cascaded in groups of 15 and are connected to a PCDU. It is not necessary for the groups to contain 15 units, and any number form 1 to 15 is supported. The CAVIS inventory system increases the number of concentrators as storage requirements expand. The inventory system can start operation with only one sensor concentrator, and additional units are added as needed. The data are collected from the concentrator units by an SPCS. ${ }^{1}$ The SPCS will support from 1 to 120 sensor concentrators connected to 8 PCDU. Larger systems must contain additional SPCS units. An inventory system with 120 concentrators can monitor 2400 items with 4800 sensors.

\subsection{POWER AND COMMUNICATION DISTRIBUTION UNIT}

For the CAVIS to be practical as a large inventory system, it must be capable of operating with the sensor concentrators distributed throughout a warehouse. This is accomplished by designing the sensor concentrators to receive power and communications over a single six-conductor cable. The PCDU is a key part of this distribution system. Fifteen sensor concentrators operating simultaneously will require up to $9 \mathrm{~A}$ of current from a $15-\mathrm{V}$ power supply. The maximum current depends on the number and type of sensors attached to the units. A group of 15 units operating on a single cable should be located within $60 \mathrm{ft}$ of the power supply. Cable lengths longer than $60 \mathrm{ft}$ will result in voltage drops that exceed the allowable limits. The PCDU supplies one-eighth of the total power requirements for a 120 -unit system. Each PCDU contains a $15-\mathrm{V}$ power supply rated at $10 \mathrm{~A}$. Distributing the power allows smaller, less expensive power supplies to substitute for one large power supply. More important is the fact that the PCDU can be strategically located to minimize cabling distance from the power source.

Another factor that must be addressed when distributing sensor concentrators is the number of RS485 loads. Guidelines for the RS485 specification state that no more than 32 loads are supported on a single drop. To increase this number, repeaters must be used. There are more recent RS485 integrated circuits that support an increased number of loads, but the CAVIS is designed to the original 32-load specification. Each PCDU provides one RS485 repeater to isolate and buffer all the SPCS nodes from the nodes connected to the PCDU. One node represents one load. Each PCDU is a node on the network branch from the SPCS. A full system of eight PCDU presents eight loads to that branch. Each sensor concentrator presents two loads to the PCDU because each processor module within the concentrator is a separate node. Up to 16 concentrators can be connected to a single PCDU before reaching the maximum of 32 loads.

The RS485 specification permits up to $1000 \mathrm{ft}$ of cable in a network branch. Greater cable lengths are supported if repeaters are used between the incremental sections of $1000 \mathrm{ft}$. The total length of cable connecting all the PCDU and the SPCS should not exceed $1000 \mathrm{ft}$. If a greater distance is necessary, additional repeaters must be installed for each increment of $1000 \mathrm{ft}$. The amount of cable in the network on the sensor concentrator side of the RS485 repeater is not an issue because the power and communications are combined into the same cable. The voltage drop requirements in the power distribution portion of the cable keep the total length well within the 1000 -ft limit. 
The PCDU is shown in Fig. 11. The unit is housed in a National Electrical Manufacturers Association (NEMA) enclosure designed to be mounted on a wall or bulkhead. Cables connected to the female connectors at the bottom of the unit supply power and communications to the sensor concentrators. Usually, a $60 \mathrm{ft}$ or less cable connects one of these connectors to the male connector on a sensor concentrator. An additional concentrator is daisychained from the female connector on this unit. This procedure is repeated until the drop contains the desired number of concentrators. Any combination of sensor concentrators can be

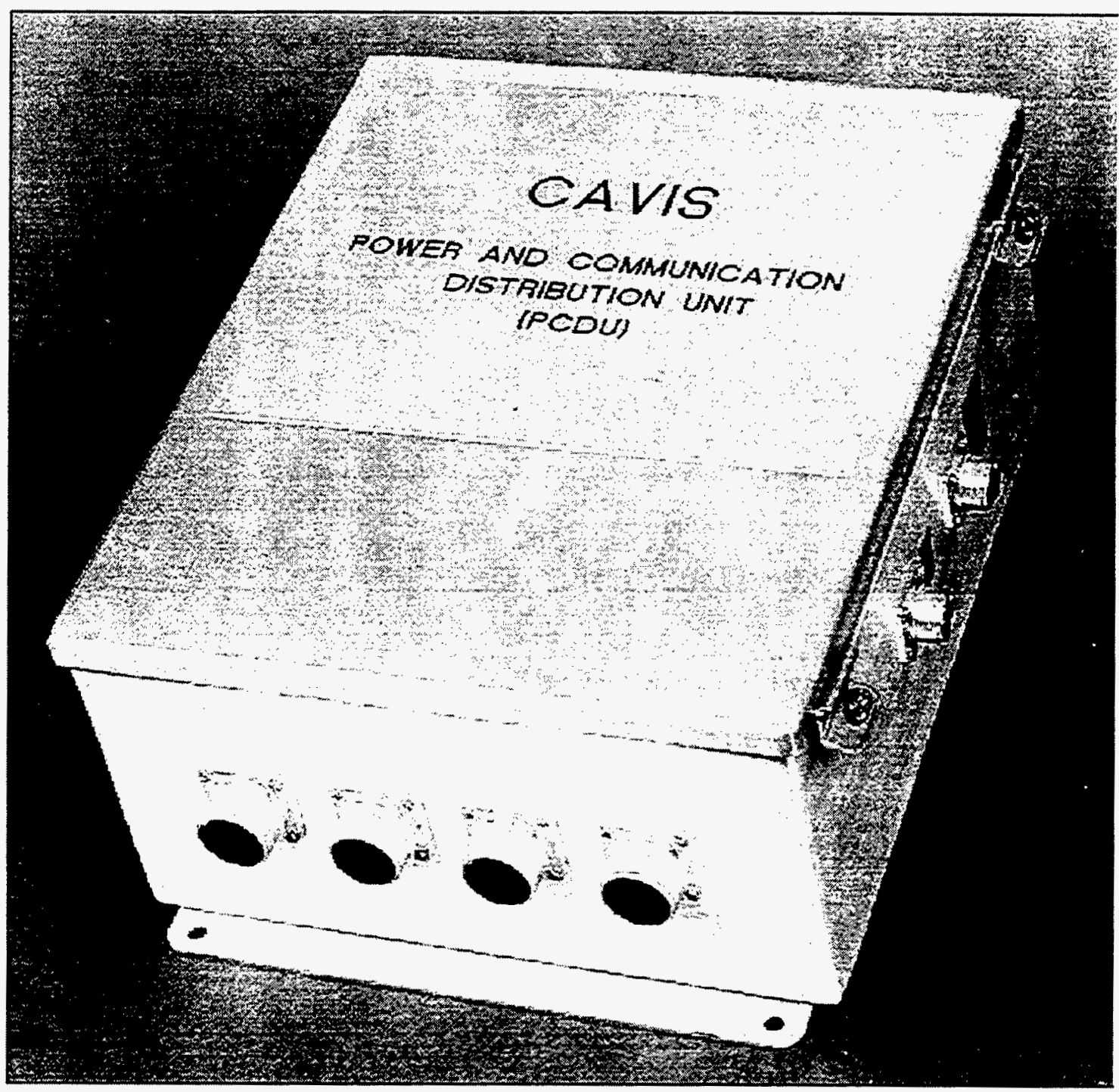

Fig. 11. The PCDU.

connected to the 4 PCDU connectors as long as the total number does not exceed 15 . If 1 cable drop contains 15 , then the other 3 connectors must remain unused. The two circular connectors on the right side of the PCDU connect the unit to the SPCS branch of the RS485 network. The male connector on the first PCDU connects directly to the RS485 cable coming from the SPCS. Additional PCDU are cascaded from the female connector. The $110-\mathrm{V}$ AC power input is located on the left side and not shown in the figure. 


\section{SENSOR MODULE INTERFACE REQUIERMENTS}

Sensor interface modules are custom designed for a particular sensor type and support 10 sensors per module. This section outlines the requirements a sensor interface module must meet to function in the sensor concentrator. Figure 12 is a block diagram of the input and output requirements for a sensor module interface. The interface to the processor module, backplane connections, and available number of sensor connections are defined and must be adhered to. A sensor interface module must condition sensor signals and convert the readings to a form usable by the processor module. Once converted, the signals are transmitted, normally upon request, to the processor module via the serial peripheral bus (SPB) portion of the module interface. All

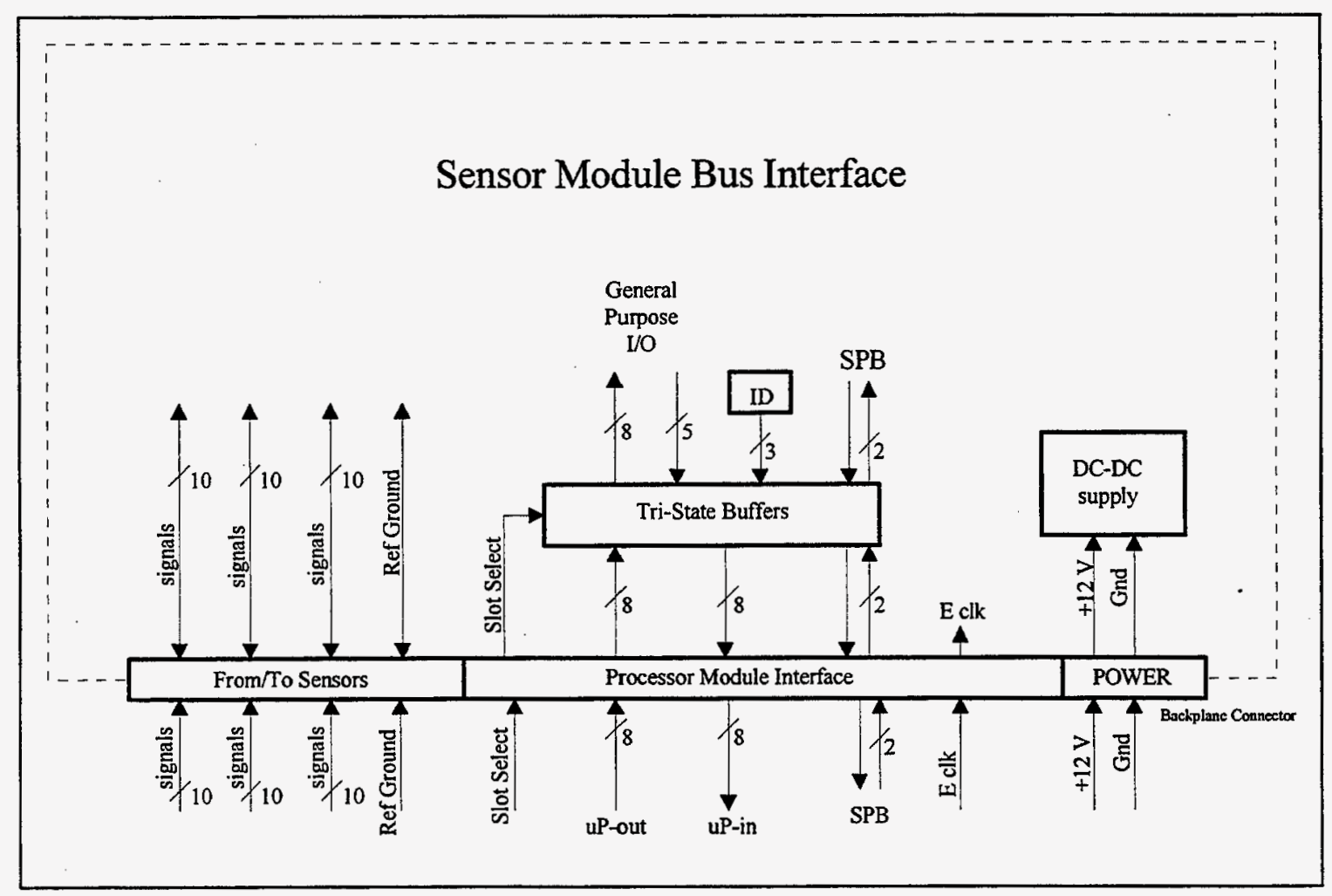

Fig. 12. Sensor module bus interface diagram.

sensor connections are made to the module from the sensor input panel through the backplane. The module must provide all the power requirements for the sensors by regulating the backplane power with on-board regulators. All connections to the module must be made through the backplane connector. Through this connector the module has access to the sensor input panel, the processor module, and the backplane power.

\subsection{THE BACKPLANE CONNECTOR}

The backplane connector is a 96-pin standard DIN size right-angle pin assembly connector (AMP 650473-5 or equivalent). The connector has 3 rows of pins each with 32 
positions. The pin "out" definitions are given in Table 1. The blank positions have no connections.

Table 1. Backplane connector pin definitions

\begin{tabular}{|c|c|c|c|}
\hline Pos & Row A & Row B & Row C \\
\hline 1 & Common power ground & Common power ground & Common power ground \\
\hline 2 & $+12 \mathrm{Vdc}$ & $+12 \mathrm{~V} \mathrm{dc}$ & $+12 \mathrm{~V} \mathrm{dc}$ \\
\hline 3 & & E-Clock & \\
\hline 4 & SPI Bus - MISO & & SPI Bus - MOSI \\
\hline 5 & & SPI Bus - Clock & \\
\hline$\overline{6}$ & uP IN 0 & uP IN 1 & uP IN 2 \\
\hline 7 & uP IN 3 & uP IN 4 & uP IN 5 \\
\hline 8 & uP IN 6 & uP IN 7 & uP OUT 0 \\
\hline 9 & uP OUT l & uP OUT 2 & uP OUT 3 \\
\hline 10 & uP OUT 4 & uP OUT 5 & uP OUT 6 \\
\hline 11 & uP OUT 7 & Slot select & \\
\hline \multicolumn{4}{|l|}{12} \\
\hline 13 & Sensor signal A0 & Sensor signal B0 & Sensor signal $\mathrm{C} 0$ \\
\hline 14 & Sensor signal A1 & Sensor signal B1 & Sensor signal $\mathrm{Cl}$ \\
\hline \multicolumn{4}{|l|}{15} \\
\hline 16 & Sensor signal A2 & Sensor signal B2 & Sensor signal C2 \\
\hline 17 & Sensor signal A3 & Sensor signal B3 & Sensor signal C3 \\
\hline \multicolumn{4}{|l|}{18} \\
\hline 19 & Sensor signal A4 & Sensor signal B4 & Sensor signal C4 \\
\hline 20 & Sensor signal A5 & Sensor signal B5 & Sensor signal C5 \\
\hline \multicolumn{4}{|l|}{21} \\
\hline 22 & Sensor signal A6 & Sensor signal B6 & Sensor signal C6 \\
\hline 23 & Sensor signal A7 & Sensor signal B7 & Sensor signal C7 \\
\hline \multicolumn{4}{|l|}{24} \\
\hline 25 & Sensor signal A8 & Sensor signal B8 & Sensor signal C8 \\
\hline 26 & Sensor signal A9 & Sensor signal B9 & Sensor signal C9 \\
\hline \multicolumn{4}{|l|}{27} \\
\hline 28 & Sensor comm. ref. signal & Sensor comm. ref. signal & Sensor comm. ref. signal \\
\hline \multicolumn{4}{|c|}{ 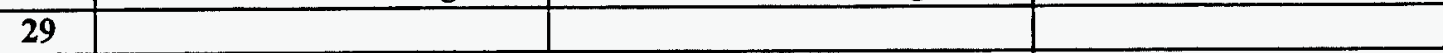 } \\
\hline 30 & Reserved & Reserved & \\
\hline 31 & Reserved & & Reserved \\
\hline 32 & Reserved & & Reserved \\
\hline
\end{tabular}

\subsection{PHYSICAL DIMENSIONS}

The module must adhere to the dimensions shown in Fig. 13. 


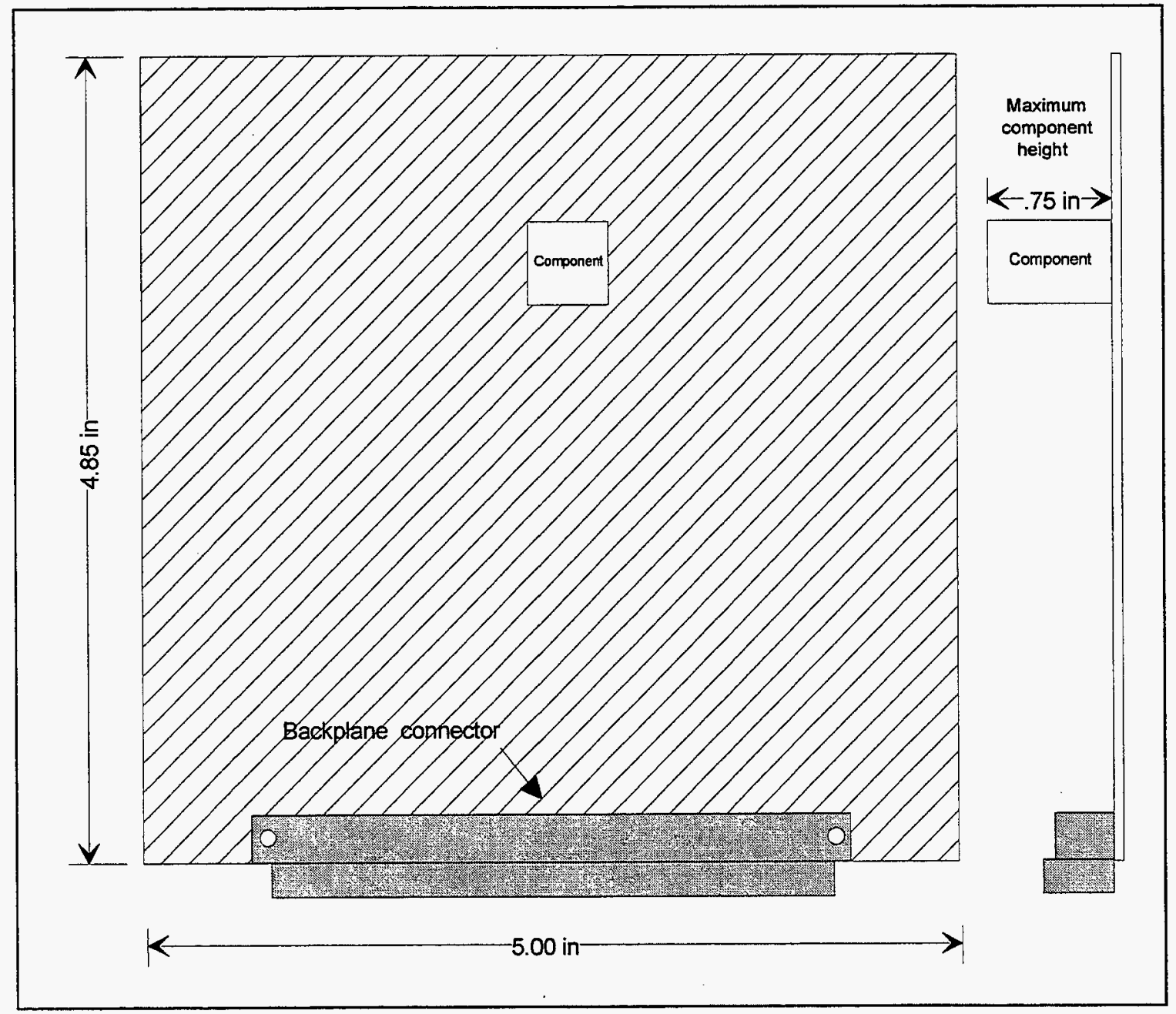

Fig. 13. Sensor module dimension requirements.

\subsection{SENSOR INPUTS}

The backplane provides 30 individual connections labeled sensor signals $\mathrm{A} 0$ to $\mathrm{A} 9, \mathrm{~B} 0$ to $\mathrm{B} 9$, and $\mathrm{C} 0$ to $\mathrm{C} 9$ from each sensor module to the sensor input panel. Each of the 10 sensors is allotted 3 of these individual lines and 1 common line. The common is a ground reference and cannot be used for any other purpose. The three individual lines may be used as inputs, outputs, or to supply power to the sensor.

The sensor module signal lines are labeled $\mathrm{Ax}, \mathrm{Bx}$, and $\mathrm{Cx}$, where $\mathrm{x}$ is the sensor position 0 to 9 . Signals A0 to A9 occupy backplane connector pin positions A13, A14, A16, A17, A19, A20, A22, A23, A25, and A26. Signals B0 to B9 occupy positions B13, B14, B16, $\mathrm{B} 17, \mathrm{~B} 19, \mathrm{~B} 20, \mathrm{~B} 22, \mathrm{~B} 23, \mathrm{~B} 25$, and B26; signals $\mathrm{C} 0$ to $\mathrm{C} 9$ occupy positions $\mathrm{C} 13, \mathrm{C} 14, \mathrm{C} 16$, $\mathrm{C} 17, \mathrm{C} 19, \mathrm{C} 20, \mathrm{C} 22, \mathrm{C} 23, \mathrm{C} 25$, and $\mathrm{C} 26$. The reference ground occupies positions $\mathrm{A} 28, \mathrm{~B} 28$, and $\mathrm{C} 28$ of the backplane connector. Note, on the sensor input panel the signal lines are labeled according to which sensor module slot on the backplane they are connected to. The outside module positions (the slots closest to the sensor input panel) are labeled $\mathrm{Ax}, \mathrm{Bx}$, and $\mathrm{Cx}$ and the middle slots (the slots next to the processor module) are labeled Dx, Ex, and Fx. Refer to Fig. 3 
and Fig. 9 for more information on slot location and how the slots relate to the two sensor input panels.

\subsection{POWER SUPPLY}

The sensor concentrator is provided with $+12 \mathrm{~V}$ dc and power ground through the system cable. The voltage will vary between 11 and 14, depending on the voltage drop across the cable. This source of power is made available to each module position through the backplane. The ground occupies positions $\mathrm{A} 1, \mathrm{~B} 1$, and $\mathrm{C} 1$ of Table 1 and positions $\mathrm{A} 2, \mathrm{~B} 2$, and $\mathrm{C} 2$ supply the $+12 \mathrm{~V}$. All module power requirements must be derived from this power using voltage regulators. Modules should not use the $+12 \mathrm{~V}$ directly because specifications allow this voltage to vary between 11 and $14 \mathrm{~V}$. Voltage regulators require an input voltage several volts higher than the regulated output voltage for correct operation which limits the highest on-module voltage to $+9 \mathrm{~V}$ when using voltage regulators. If voltages higher than $9 \mathrm{~V}$ or negative voltages are necessary, then dc-to-dc converters must be used. Each sensor module is permitted to draw a maximum of $150 \mathrm{~mA}$ from the $12 \mathrm{~V}$ supply. As a minimum, each sensor module must generate $+5 \mathrm{~V}$ to power the buffers, ID generation logic, and the SPB interface of Fig. 12 .

\subsection{PROCESSOR MODULE INTERFACE}

The interface with the processor module consists of the following sensor module input and output connections through the backplane connector (Refer to Fig. 12).

- E-clock (unbuffered),

- slot select (unbuffered),

- sensor module identification (ID) ( three digital output lines - all buffered),

- the SPB bus (two digital input lines and one digital output line - all buffered), and

- general purpose I/O (eight digital input lines and five digital output lines - all buffered).

\subsubsection{E-clock}

The E-clock is generated by the processor module microprocessor at a frequency of $1.8432 \mathrm{MHz}$ and is always available. The E-clock is assigned position B3 in the backplane connector.

\subsubsection{Slot Select}

The slot select is used by the processor module to enable communications to/from that particular slot. The slot select enables the buffers for the serial peripheral interface (SPI) bus, the ID, and other digital input/output (I/O) lines. The slot select signal is assigned to individual slot positions at pin $\mathrm{B} 11$ in the backplane connector. The $\mathrm{B} 11$ pin positions are not bused together, and when the module is inserted into different slots it will receive a specific slot select for that position.

\subsubsection{Sensor Type ID}

Each sensor module must provide a unique type ID enabling the processor module to identify the sensor type being supported. The processor module uses the type ID to select the correct data- 
gathering routine for the sensor module. The ID consists of three logic states permanently wired to ground or pulled up to $+5 \mathrm{~V}$ with resistors. The following IDs are identified:

0 RAD-COUPLE gamma sensor,

1 RAD-SIP gamma sensor,

2 FIB-WT rubber fiber optic weight sensor,

3 CAP-WT capacitive weight and temperature sensor,

$4 \quad$ FIB-GAM fiber optic gamma sensor, and

7 no module present.

The ID bits are buffered and driven onto the backplane when the processor module activates the slot select line. The ID propagates to the backplane as signals uP-IN0 (ID LSB), uP-IN1, and uP-IN2 (ID MSB) at positions A6, B6, and C6, respectively.

\subsubsection{Serial Peripheral Bus}

The SPB is based on the Motorola SPI. This bus provides the mechanism for exchanging data and sending control signals between the processor module and the sensor module. The SPI bus consists of three signals: a clock that synchronizes all the communications, the Master Out Slave In (MOSI) data line, which carries data from the microprocessor to the device, and the Master In - Slave Out (MISO), which carries data from the device to the microprocessor. Transmission in both directions occurs simultaneously, for example, the data in and out for master or slave are clocked with the same clock edges. The sensor module SPI signals are only active to the backplane when the module slot select is enabled. The SPI clock occupies position B5 on the backplane connector. The MOSI data line is at position $\mathrm{C4}$, and the MISO data line is at position $\mathrm{A} 4$.

\subsubsection{General Purpose $\mathrm{I} / \mathrm{O}$}

A number of sensor module connections to the backplane connector are designated as general purpose I/O. There are eight sensor module digital input lines and five sensor module digital output lines. All of these lines are buffered and propagate to and from the backplane connector only when the module slot select signal is active. The designer can use these lines as chip selects for devices on the sensor module or for handshaking and control purposes. The general purpose sensor module input lines occupy positions B8, A9, B9, C9, A10, B10, C10, and A11 (uP-OUT0 to uP-OUT7, respectively) on the backplane connector. The general purpose sensor module output lines occupy positions A7, B7, C7, A8, and B8 (uP-IN3 to uP-IN7, respectively) on the backplane connector.

\subsection{ALTERA FLEX 8000}

If the sensor module design includes an Altera Flex 8000 programmable logic device, the module design should adhere to the following guidelines to take advantage of the firmware utilities built into the processor module.

\subsubsection{Microprocessor Altera Program Load}

The Altera Flex 8000 circuit should be designed with the capability of loading the internal program by one of two methods. One method should allow that the program be loaded from a serial EPROM installed in a socket on the module. The other method should allow for the 
program to be loaded by the microprocessor on the processor module via the SPB. The method used should be selected by jumpers on the board sensor module.

When loaded by the microprocessor, the following signals should be connected:

- bus uP-OUT7 (buffered) to the NCONFIG pin of the Flex 8000 ,

- bus SPI-MOSI (buffered) to the +DATA0 pin of the Flex 8000,

- bus SPI_CLK (buffered) to DCLK pin of the Flex 8000 ,

- bus uP-IN7 (buffered) to NSTATUS pin of the Flex 8000, and

- bus uP-IN6 (buffered) to CONF_DONE pin of the Flex 8000.

\subsubsection{Software Access to the Altera Flex 8000}

The normal chip select for accessing the Altera Flex 8000 is uP-OUT0. The uP-OUT7 is used only for internal program loading. 


\section{PROCESSOR MODULE FIRMWARE}

Other Documents. The "CAVIS Sensor Bus Protocol and Commands" is referenced for sections related to serial communications and is included in this report as Appendix A. In the current Continuous Automated Vault Inventory System (CAVIS) design, the format of the data packet has been modified and additional commands have been added. All other information contained in Appendix A is still relevant.

\subsection{DESCRIPTION}

The processor module firmware performs tasks that acquire sensor data from the sensor modules and that communicate with the main data collection computer. The main data collection computer is referred to as the Sensor Polling and Configuration System (SPCS). This computer sends commands to and collects data from the processor modules. One SPCS can manage up to 240 processor modules. The processor modules are usually grouped as pairs and housed within a sensor concentrator. During initialization the processor module determines the type of each of the two sensor modules it is attached to. The firmware contains the data acquisition routines for all defined sensor modules. The appropriate routine to use with each sensor module is selected based on the sensor module types detected. After hardware and variable initialization, the firmware enters a loop where it continuously tests software flags to determine the next task to perform. These flags are set by the serial communications interrupt and the timer interrupt service routines. The communications interrupt indicates a new command message has been received from the SPCS, and the timer interrupt schedules the next data acquisition cycle from the associated sensor modules.

When the communication flag is set, the incoming message is processed and the command decoded. The service required by the command is performed, and an appropriate response message is transmitted back to the SPCS. Commands fall into one of four groups: configuration commands, data acquisition commands, diagnostics commands, and setup commands. These commands are described Sect. 6.7.

When the timer flag is set, a data acquisition cycle is performed for one or more of the sensor channels. The timer flag ensures that data from a sensor are gathered at a frequency sufficient to support integration and signal averaging. Different sensors may have different sampling rate requirements, and there can be multiple timer flags to accommodate each sensor.

Each processor module is uniquely identified by a 48-bit ID number embedded in the internal EEPROM of the microprocessor. The 8-bit CAVIS sensor bus node address for the processor module is also stored in the internal EEPROM. The setting of these registers is enabled only under specific authorized installation conditions while the module is disconnected from the SPCS. Write access to these registers is prevented during normal operation.

\subsection{INITIALIZATION}

The initialization process is entered when the module is powered up or the microprocessor is reset with the manual switch. For explanation of the following microprocessor hardware terminology, refer to the Motorola 68HC11F1 Technical Manual.

\subsubsection{Microprocessor Hardware Configuration}


The microprocessor hardware devices I/O Ports, SCI, and SPI are initialized from configuration values stored in program memory.

\subsubsection{Input/Output Ports}

The first task performed is the initialization of internal registers and $\mathrm{I} / \mathrm{O}$ ports. For each bidirectional port, a direction register mask is set to specify whether the $\mathrm{I} / \mathrm{O}$ pins are inputs or outputs.

\subsubsection{Serial Communication Interface}

The SCI port register is set to the default communication parameters. The current default configuration is 9600 baud, 8 bits, no parity, and the receive interrupt enabled.

\subsubsection{Serial Peripheral Interface}

The SPI default configuration is set to accept data on the rising edge of the clock, and the SPI clock is set to the maximum rate (in this implementation, $921.6 \mathrm{kHz}$ ). This rate can be changed programmatically by individual sensor data acquisition routines if necessary.

\subsubsection{Node Configuration}

After the hardware ports are initialized the processor module determines the configuration of its node, for example, if the node is odd or even and the type, if any, of the sensor modules in "Position-A" and "Position-B".

\subsubsection{Odd/Even}

The odd/even parameter is determined by the backplane when the processor module is inserted. The microprocessor determines this parameter by reading bit zero of port $\mathrm{G}$. If the bit is at a logic zero the processor module is assigned the even node address; otherwise, it is assigned the odd node address. Processor modules are programmed only with even node addresses, and the odd addresses are not assigned. If the processor module is located in an odd position, the firmware adds one to the even address to determine the actual address that the module will respond to. Whether the node address is odd or even is used by the SPCS to associate the physical location of the sensors. Processor modules with odd addresses return readings from sensor modules installed in slots 1 and 3. Likewise, processor modules with even addresses return readings from sensor modules installed in slots 2 and 4 . Refer to Sect. 2.6 for further explanation.

\subsubsection{Sensor Module Type.}

The determination of the two sensor module types associated with the processor module is performed by selecting each slot and reading the sensor module ID. The term "Position-A" is defined as slot 1 on the odd side and as slot 4 on the even side. The term "Position-B" is defined as slot 3 on the odd side and as slot 2 on the even side. Currently, six of the eight possible sensor module types are defined as follows:

$0=$ RAD-COUPLE gamma sensor

$1=$ RAD-SIP gamma sensor

$2=$ FIB-WT rubber fiber-optic weight sensor

$3=$ CAP-WT capacitive weight and temperature sensor

$4=$ FIB-GAM fiber optic gamma sensor

$7=$ No module present 


\subsubsection{Sensor Data Arrays and Variables}

A generic approach is used to identify sensor data arrays and variables since more than two different types of sensor modules can be supported by a single version of firmware. Because only two sensor modules are supported simultaneously, only two sets of sensor data and variables are required in the firmware. These data and variables are implemented as "Position- $A$ " sensors 1 through 10 and "Position-B" sensors 1 through 10. The data and variables are stored in arrays whose first dimension identifies the sensor module position. A value of zero indicates "Position$A$ " ownership, and a value of one indicates "Position-B" ownership. Some sensors, such as the capacitive weight sensor, measure two parameters. This sensor measures both weight and temperature. To accommodate two parameter sensors, a duplicate set of data arrays and variables is provided for the second parameter. All data arrays and variables are of integer type (two bytes for each entry) and array index pointers are of character type (one byte for each entry). The arrays and array indexes are initialized to zero, and the array size to the predefined setting for the sensor type.

\subsubsection{Communication Data Buffers and Variables}

The communication data buffers and variables referred to in the following are explained further in Appendix A, "CAVIS Sensor Bus Protocol and Commands." The characters received by the serial communication interface are stored in a wraparound buffer structure named rcv. This structure consists of $\mathbf{n}$ pkts (default 4) of packet array buffers of size pkt_size (default 100 bytes), two buffer pointer variables, one to the next available buffer (nxt) and one to the current buffer (cur), and a character count (charent) variable for the current buffer. Characters are transmitted via the output buffer sendBuffer of length pkt_size (default 100 bytes). All these buffer variables and pointers are initialized to zero at reset.

\subsection{MAIN LOOP}

The main firmware loop consists of testing for task flags that are set to TRUE and executing any indicated tasks. There can be as many as five active task flags, one flag for communications and two flags per sensor module. All flags are initialized to FALSE.

\subsubsection{Communication Task Flag}

The communication flag is the value returned by the packetReady 0 function and when TRUE indicates that a complete packet has been received and needs processing.

\subsubsection{Sensor Timer Task Flags}

The sensor flags indicate that a predefined time has expired and that a data acquisition cycle should be performed. Some sensors require different processing at different time intervals, thus requiring multiple flags to complete acquisition. There are two flags allocated for each sensor, and all unused flags are set to FALSE.

\subsection{SERIAL COMMUNICATIONS}

The serial communications follow in general the protocol established in the earlier generation of the sensor concentrator (that protocol is described in Appendix A). Changes have been made to accommodate the expanded command structure and related node responses. 


\subsubsection{Serial Interrupt Service Routine}

The serial interrupt service routine is invoked when the SCI of the microprocessor receives a character. The interrupt is generated after the character is transferred to the receive register. The interrupt service routine reads the character from this register and stores it into the current packet buffer. The service routine also tests for the valid start of a packet and fills the packet buffer until the expected character count or the default packet size or is reached. The expected character is given in the fourth byte of the packet. Once a complete packet is received a flag is set and the interrupt service routine terminates.

\subsubsection{Receiving messages}

The program tests the flag returned by the packetReady function to detect when the service routine has received a complete packet. Once the packet is detected it is processed to check its integrity and node address. If the packet does not contain errors and the node address matches the address of the processor module, the message command is decoded and the indicated task performed. The routines testPacket() and processPacket() are invoked to test and process packets.

\subsubsection{Test Packet Routine}

The test packet routine first checks for a valid pattern at the end of the packet. Note that the serial interrupt service routine tested for the correct packet header. If the packet tail is correct a simple, one byte checksum is calculated by adding all the bytes of the packet ignoring the carries. The calculated checksum for the packet is compared to the received checksum byte, and if a match occurs the packet is flagged as good. If at anytime during the execution of the test packet routine an error is found, the routine returns with an error code.

\subsubsection{Process Packet Routine}

The Process Packet routine first checks whether the received command is address dependent. If it is, then the message address is compared to the node address. If the two addresses compare, the command is executed; otherwise, the command is ignored. Address-independent commands are always executed.

\subsubsection{Transmitting messages}

The transmission of messages, normally a response to a command, is a two-step process. First, the specific command routine fills the SendBuffer with the appropriate data. Then the send buffer routine is executed to transmit the message via the serial communications interface.

\subsection{SENSOR DATA ACQUISITION}

\subsubsection{RAD-SIP Gamma Sensor}

The RAD-SIP gamma sensor measurement consists of counting and reporting the number of $185.7 \mathrm{keV}$ gamma pulses detected during a specific time interval. The pulse output from each sensor discriminator circuit is connected to a dedicated counter. The counters are located in a programmable logic device (PLD). At a predetermined periodic rate, the counters are latched and read by the microprocessor via the SPI. Immediately after the values in the counters are latched, the counters are cleared and a new count period is started. The count values are 
accumulated in registers within the microprocessor until the accumulated value represents the total count for 1 second. The number of accumulations required depends on the time interval of the counters. For example, counter intervals of $250 \mathrm{~ms}$ would require four accumulations to equal 1 second. Each 1 second count interval is stored in an array of a predefined length. The oldest value is dropped from the array, and the most recent 1 second interval is entered into the array. When the SPCS requests data from the sensor board, the values in the array are summed and the results are divided by one-tenth of the array length. The results are that the reported value represents the current count average with a one-tenth count resolution.

\subsubsection{Initialization.}

The software initializes the size of the averaging array, the intervals for the timer interrupt, and the appropriate temporary registers. The hardware counters contained in the PLD are also cleared. This sensor is currently the only sensor that uses two timer intervals. One timer interval must be an exact submultiple of the other and is used to coordinate the execution of additional software processing. Since the timer ticks 225 times a second, one of the interval timers is set to 225 (one event per second), and the other to 25 (nine events per second).

\subsubsection{Reading data.}

There are two processes associated with gathering RAD-SIP data. The first occurs nine times per second, and consists of reading the value of each counter and adding the count to a temporary register. After nine accumulations the second process is executed to store the accumulated value into the averaging array. The temporary register is then cleared to zero, and the process is repeated. The 1 -second accumulated readings are continuously entered into the averaging array with the oldest value discarded each time. The average is calculated only when the data collection system requests the data.

\subsection{CAPACITIVE WEIGHT PAD AND TEMPERATURE SENSOR}

The Capacitive Weight Pad measures both weight and temperature. The value of both parameters is encoded into a single periodic signal. The period of the signal represents temperature, and the pulse width represents weight. The period varies between 1 and $3 \mathrm{~ms}$ and the pulse width between 200 and $300 \mu$ s. The capacitive weight sensor module PLD measures both the period and pulse width of the signal by accumulating counts of the on-board $10-\mathrm{MHz}$ clock. The resulting counts are then read by the processor module.

\subsubsection{Reading Data}

To perform a measurement the power to two weight sensors is turned on. Only two sensors at a time are turned on to conserve power. After a short delay to allow the circuitry to settle, the counters for the temperature (period) and weight (pulse) are read. To reduce the possibility of crosstalk, nonadjacent channels are powered on together. To make the waiting time more efficient, the two channels that are powered on are read as opposed to reading channels in sequential order. The following sequence is repeated continuously to collect weight information (initially the power is on for the first pair of sensors):

1. Read the first channel of the pair.

2. Read the second channel of the pair. 
3. Turn power on to the next pair of channels (power is automatically turned off for the previous pair).

This sequence is performed three times each second, allowing approximately $330 \mathrm{~ms}$ after the power is applied for the sensor circuitry to settle. Approximately 1.5 seconds is required to collect a reading from all ten sensors connected to the interface module. The collected sensor readings are stored in an averaging array by the processor module. The processor module retains readings for the last 30 seconds and discards the oldest reading. The actual averaging of the data is performed only when the SPCS requests the weight sensor data.

\subsection{COMMANDS AND RESPONSES}

The communications commands and responses follow the general rules of the CAVIS protocol described in Appendix A. The command packets initiated by the SPCS conform to the format contained in Table 2.

Table 2. Command packet format

\begin{tabular}{|c|c|c|}
\hline Byte position & Value(s) & Description \\
\hline $0-2$ & STX $(0 \times 02)$ & Start pattern - 3 bytes, each an STX \\
\hline 3 & $10-255$ & $\begin{array}{l}\text { Byte count in packet including start and stop } \\
\text { pattern and checksum }\end{array}$ \\
\hline 4 & $2-241,255$ & $\begin{array}{l}\text { Destination node addresses } 2-241 \text { are valid } \\
\text { addresses, } 255 \text { is the default address for } \\
\text { unconfigured nodes and is used with address } \\
\text { independent commands }\end{array}$ \\
\hline 5 & $0-255$ & Command code \\
\hline $6-n$ & (Command dependent) & $\begin{array}{l}0 \text { to }(n-6) \text { bytes of parameters associated with } \\
\text { the command }\end{array}$ \\
\hline$n+1-n+3$ & $\operatorname{ETX}(0 \times 03)$ & Stop pattern - 3 bytes, each an ETX \\
\hline$n+4$ & $0-255$ & $\begin{array}{l}\text { 8-bit checksum of all bytes in the packet (except } \\
\text { checksum) }\end{array}$ \\
\hline
\end{tabular}

For every valid command there will be a response from the node conforming to the general format given in Table 3. 
Table 3. Response packet format

\begin{tabular}{|c|c|c|}
\hline Byte position & Value(s) & Description \\
\hline$\overline{0-2}$ & STX $(0 \times 02)$ & Start pattern - 3 bytes, each an STX \\
\hline 3 & $10-255$ & $\begin{array}{l}\text { Byte count in packet including start and stop } \\
\text { pattern and checksum }\end{array}$ \\
\hline 4 & 0 & Destination address \\
\hline 5 & $2-241,255$ & $\begin{array}{l}\text { Source node addresses } 2-241 \text { are valid addresses, } \\
255 \text { is the default address for unconfigured nodes } \\
\text { and is used with address-independent commands }\end{array}$ \\
\hline 6 & $0-1$ & $\begin{array}{l}0=\text { first message since reset } \\
1=\text { normal message }\end{array}$ \\
\hline $7-8$ & $0-65535$ & Incremental message number \\
\hline 9 & Bit related & $\begin{array}{l}\text { Master error code, } 0=\text { no error } \\
\text { bit } 0=\text { "Position-A" error } \\
\text { bit } 1=\text { "Position- } \mathrm{B} " \text { error } \\
\text { bit } 2=\text { serial communication error } \\
\text { bit } 3=\text { invalid command }\end{array}$ \\
\hline $10-n$ & (Response dependent) & DATA \\
\hline$n+1-n+3$ & $\operatorname{ETX}(0 \times 03)$ & Stop pattern - 3 bytes, each an ETX \\
\hline $\mathrm{n}+4$ & $0-255$ & $\begin{array}{l}\text { 8-bit checksum of all bytes in the packet (except } \\
\text { checksum) }\end{array}$ \\
\hline
\end{tabular}

Table 4 is a summary of the command codes. The command code is inserted into the command packet at byte position 6 . The data for commands that have parameters follow byte position 6 .

Table 4. Summary of command codes

\begin{tabular}{clll}
\hline $\begin{array}{c}\text { Code } \\
\text { (hex) }\end{array}$ & Command & $\begin{array}{c}\text { Additional } \\
\text { parameters }\end{array}$ & Comments \\
\hline & Data and status & Reserved \\
\hline 00 & & Reserved \\
01 & & $\begin{array}{l}\text { Exception counter is } \\
\text { cleared after reading }\end{array}$ \\
\hline
\end{tabular}


04 Node configuration

05 Send "Report-A"

06 Send "Report-B"

07-3F

Not defined

\section{Configuration}

40

41

42 Read configuration parameters
Not defined
Reserved

Reserved

43-7F

\section{Diagnostics}

\begin{tabular}{clll}
\hline 80 & Read sensor type ID & Slot & \\
81 & Verify sensor PLD circuit & Slot, value & \\
82 & Send sensor data & Slot, channel & \\
83 & & & \\
84 & Reinitialize all & & Not defined \\
$85-\mathrm{BF}$ & & & \\
& & & \\
\hline $\mathrm{C} 0$ & Read address & Side & \\
$\mathrm{C} 1$ & Set address & Side, address & \\
$\mathrm{C} 2$ & Set 48-bit serial ID & Side, ID, key & \\
C3-FF & & & Not defined \\
\hline
\end{tabular}

When a node receives an invalid command or parameter, the "Invalid Command" error code is set in the node error variable and a response message is sent to the SPCS. The invalid command or parameter response is shown in Table 5. 
Table 5. Invalid command response

\begin{tabular}{lll}
\hline Byte position & Value(s) & Description \\
\hline 10 & $0-\mathrm{FF}$ & Returns the command code \\
11 & $\begin{array}{l}0 \times 80 \\
0 \times 8 \mathrm{X}\end{array}$ & $\begin{array}{l}\text { Commands without parameters, } \\
\text { where } \mathrm{X} \text { is the parameter in error for commands with } \\
\text { parameters (i.e., 0x82 if error detected in } 2^{\text {nd }} \text { parameter) }\end{array}$ \\
\hline
\end{tabular}

\subsubsection{Command Descriptions}

This section lists the commands by "Command Code" and follows each code with an

"Additional Parameters" section and a "Response Data" section. Most of the commands do not require additional parameters. Command codes that are not defined or used are identified.

0x00 Code not used

0x01 Send Report (not used)

$0 \times 02$ Status

No additional parameters required.

Table 6. Status command response data

\begin{tabular}{lll}
\hline Byte position & Value(s) & Description \\
\hline 10 & 0,1 & Side \\
11 & $0-255$ & Value of exception counter (cleared after this command) \\
12 & & "Position-A" status/error code (see section 6.8) \\
13 & 0,4 & "Position-B" status/error code (see section 6.8) \\
14 & 0,4 & Status of PLD in "Position-A" $(0=$ ok, $4=$ no program) \\
15 & 0,1 & Serial ID set flag ( $0=$ ID not set, $1=$ ID set $)$ \\
16 & 0,1 & Node address set flag $(0=$ not set, $1=$ set $)$ \\
17 & 0,1 & EEPROM status ( $0=$ unprotected, $1=$ protected $)$ \\
18 &
\end{tabular}

0x03 Query Node (not used) 


\section{0x04 Configuration (brief)}

No additional parameters required.

Table 7. Configuration command response data

\begin{tabular}{lll}
\hline Byte position & Value(s) & Description \\
\hline 10 & 0,1 & Side \\
$11-16$ & $0-\left(2^{48-2)}\right.$ & Processor module ID \\
17 & $0-7$ & "Position-A" module type (see Table 25) \\
18 & $0-7$ & "Position-B" module type (see Table 25) \\
19 & $1-10$ & Number of channels \\
\hline
\end{tabular}

\section{0x05 Send Report A}

0x06 Send Report B

No additional parameters required.

Table 8. Send Report A / Send Report B response data

\begin{tabular}{|c|c|c|}
\hline Byte position & Value(s) & Description \\
\hline 10 & $0-255$ & Slot error/status (see section 6.8) \\
\hline 11 & $0-7$ & Module type ID (see Table 25) \\
\hline 12 & 0,1 & $0=$ one parameter, $1=$ two parameters \\
\hline $13-32$ & & $\begin{array}{l}10 \text { unsigned integer values representing data from the first } \\
\text { parameter }\end{array}$ \\
\hline $33-52$ & & $\begin{array}{l}10 \text { unsigned integer values representing data from the second } \\
\text { parameter (if present) }\end{array}$ \\
\hline
\end{tabular}

0x07-0x3F Codes not used

Ox40 Reserved

0x41 Reserved

0x42 Read Complete Configuration

No additional parameters required. 
Table 9. Read Complete Configuration command response data

\begin{tabular}{lll}
\hline Byte position & Value(s) & Description \\
\hline $10-15$ & $0-\left(2^{48-2)}\right.$ & Processor module ID \\
16 & & Side \\
& & Serial baud rate code \\
17 & & SPI setup code \\
18 & Number of channels \\
19 & Maximum array size allowed \\
& \\
$20-22$ & "Position-A" module type (see Table 25) \\
23 & Array upper limit parameter 1 \\
$24-26$ & Array upper limit parameter 2 \\
$27-29$ & Timers \\
$30-32$ & "Position-B" module type (see Table 25) \\
33 & Array upper limit parameter 1 \\
$34-36$ & Array upper limit parameter 2 \\
$37-39$ & Timers \\
$40-42$ & & \\
\hline
\end{tabular}

0x43-0x7F Codes not used

0x80 Read Sensor Type ID

Table 10. Read Sensor Type ID command parameters

\begin{tabular}{lll}
\hline Byte position & Value(s) & Description \\
\hline 6 & $0-1$ & Slot; $0=A, 1=B$ \\
\hline
\end{tabular}

Table 11. Read Sensor Type ID response data

\begin{tabular}{lll}
\hline Byte position & Value(s) & Description \\
\hline 10 & $0-1$ & Slot; $0=\mathrm{A}, \mathrm{I}=\mathrm{B}$ \\
11 & $0-7$ & Module type ID set in register \\
12 & $0-7$ & Module type ID (current reading) \\
\hline
\end{tabular}

Ox81 Verify PLD 
Table 12. Verify PLD command parameters

\begin{tabular}{lll}
\hline Byte position & Value(s) & Description \\
\hline 6 & $0-1$ & Slot position; $0=\mathrm{A}, 1=\mathrm{B}$ \\
7 & $0-255$ & Value for verification \\
\hline
\end{tabular}

Table 13. Verify PLD response data

\begin{tabular}{lll}
\hline Byte position & Value(s) & Description \\
\hline 10 & & Error code (see section 6.8) \\
11 & $0-1$ & Slot position; $0=\mathrm{A}, 1=\mathrm{B}$ \\
12 & $0-7$ & Module type ID \\
13 & $0-255$ & Value sent \\
14 & $0-255$ & Value read \\
\hline
\end{tabular}

\section{0x82 Read Single Data Channel}

Table 14. Read Single Data Channel command parameters

\begin{tabular}{lll}
\hline Byte position & Value(s) & Description \\
\hline 6 & $0-1$ & Slot position; $0=\mathrm{A}, 1=\mathrm{B}$ \\
7 & $0-9$ & Channel number \\
\hline
\end{tabular}

Table 15. Read Single Data Channel response data

\begin{tabular}{lll}
\hline Byte position & Value(s) & Description \\
\hline 0 & & Error code (see section 6.8) \\
1 & $0-1$ & Slot position; $0=\mathrm{A}, \quad$ 1 = B \\
2 & $0-7$ & Module Type ID \\
3 & $0-9$ & Channel number \\
$4-5$ & $0-65535$ & Data value (unsigned integer) parameter 1 \\
$6-7$ & $0-65535$ & Data value (unsigned integer) parameter 2 \\
\hline
\end{tabular}

0x83 Reserved 
0x84 Reinitialize ALL

No Additional Parameters required

Table 16. Reinitialize $A L L$ command response data

\begin{tabular}{lll}
\hline Byte position & Value(s) & Description \\
\hline 10 & $0-1$ & Processor module location $(0=$ even side, $1=$ odd side $)$ \\
11 & $0 \times 84$ & Reinitialize command code \\
\hline
\end{tabular}

0x85-0xBF Codes not used

0xC0 Read Node Address [NULL (255) node address]

Table 17. Read Node Address command parameters

\begin{tabular}{lll}
\hline Byte position & Value(s) & Description \\
\hline 6 & $0-1$ & Processor module location $(0=$ even side, $1=$ odd side $)$ \\
\hline
\end{tabular}

Table 18. Read Node Address response data

\begin{tabular}{lll}
\hline Byte position & Value(s) & Description \\
\hline 0 & $0-1$ & Processor module location $(0=$ even side, $1=$ odd side $)$ \\
1 & $2-240,255$ & Node address, $255=$ unconfigured node \\
2 & $2-241,255$ & Actual node address, $255=$ unconfigured node \\
\hline
\end{tabular}

0xC1 Set Node Address [NULL (255) node address]

Table 19. Set Node Address command parameters

\begin{tabular}{lll}
\hline Byte position & Value(s) & Description \\
\hline 6 & $0-1$ & Processor module location $(0=$ even side, $1=$ odd side $)$ \\
7 & $2-240$ & Even node address \\
\hline
\end{tabular}


Table 20. Set Node Address response data

\begin{tabular}{lll}
\hline Byte position & Value(s) & Description \\
\hline 0 & & Error code (see section 6.8) \\
1 & $0-1$ & Processor module location $(0=$ even side, 1 = odd side) \\
2 & $2-240,255$ & Current node address, $255=$ unconfigured node \\
3 & $2-240,255$ & Previous node address, $255=$ unconfigured node \\
\hline
\end{tabular}

0xC2 Set Processor Module 48-bit Serial ID [NULL (255) node address]

Table 21. Set processor module 48-bit Serial ID command parameters

\begin{tabular}{lll}
\hline Byte position & Value(s) & Description \\
\hline 6 & $0-1$ & Processor module location $(0=$ even side, $1=$ odd side $)$ \\
$7-12$ & $0-\left(2^{48}-2\right)$ & Processor module ID \\
$13-14$ & $0-65535$ & Security key \\
\hline
\end{tabular}

Table 22. Set processor module 48-bit Serial ID response data

\begin{tabular}{lll}
\hline Byte position & Value(s) & Description \\
\hline 0 & & Error code (see section 6.8) \\
1 & $0-1$ & Processor module location $(0=$ even side, $1=$ odd side) \\
$2-7$ & $\begin{array}{l}0-\left(2^{48}-2\right), \\
\left(2^{48}-1\right)\end{array}$ & Current processor module ID, $\left(2^{48}-1\right)=$ unconfigured \\
\hline
\end{tabular}

0xC3-0xFF Codes not used

\subsection{MASTER ERROR CODES}

Master error codes are reported in the header of every response message from the sensor concentrator node. The codes are bit related, and multiple codes are ORed together into the same byte, for example, if both a "Position A" and a "Position B" error are present, the error code byte will contain $0 \times 03$. 
Table 23. Master error codes

\begin{tabular}{cl}
\hline Code & \multicolumn{1}{c}{ Description } \\
\hline $0 \times 00$ & No error \\
$0 \times 01$ & "Position-A" error \\
$0 \times 02$ & "Position-B" error \\
$0 \times 04$ & Serial communication error \\
$0 \times 08$ & Invalid command \\
$0 \times 10$ & Internal EEPROM \\
\hline
\end{tabular}

\subsection{STANDARD ERROR CODES}

The standard error codes, if applicable, are reported in the first byte of the DATA section of a response message. Some response messages do not include an error code. These error codes are in addition to the master error codes and specify what caused the master error. Errors are either general or board specific. The general errors relate to the overall operation and status of the electronics. Board-specific errors normally relate to errors that occur during a measurement, for example, time-out. Table 24 lists the standard error codes by priority, with the lowest priority at the top of the table. When multiple errors occur, the error with the highest priority is reported.

Table 24. Standard error codes

\begin{tabular}{cl}
\hline Code & \multicolumn{1}{c}{ Description } \\
\hline $0 \times 00$ & No error \\
$0 \times 02$ & No module present \\
$0 \times 03$ & No PLD present \\
$0 \times 04$ & PLD not programmed successfully \\
$0 \times 05$ & Internal EEPROM read error \\
$0 \times 80-0 \times 9 F$ & Invalid command parameter (0x80+parameter position) \\
$0 \times 06$ & PLD verify failure \\
$0 \times 07$ & Internal EEPROM protected \\
$0 \times 08$ & Internal EEPROM write error \\
$0 \times 20$ & Measurement time-out (processor module specific) \\
\hline
\end{tabular}


6.10. SENSOR MODULE TYPE ID

Table 25. Sensor module identification codes

\begin{tabular}{lccccc}
\hline \multicolumn{1}{c}{ Sensor module } & Type ID & \multicolumn{2}{c}{ Parameter 1 } & Parameter 2 \\
\hline RAD-COUPLE & 0 & Gamma & $0-4095$ & & \\
RAD-SIP & 1 & Gamma & $0-65535$ & & \\
FIB-WT & 2 & Weight & $0-65535$ & & \\
CAP-WT & 3 & Weight & $0-65535$ & Temp & $0-65535$ \\
FIB-GAM & 4 & Gamma & $0-65535$ & & \\
\hline
\end{tabular}




\section{REFERENCES}

1. J. J. Dunigan et al., Graphical Facility Information Center Functional System Design Document, Y/DW-1636, Lockheed Martin Energy Systems, Inc., Oak Ridge Y-12 Plant, September 1996.

2. "Serial Communications Interface," Sect. 9 in MC68HC11F1 Technical Data Reference Manual, MC68HC11F1/D, Rev. 2, Motorola, 1990.

3. C.A. Pickett et al., A Continuous Automated Vault Inventory System (CAVIS) for Accountability Monitoring of Stored Nuclear Materials, Y/DW-1351, Lockheed Martin Energy Systems, Inc., Oak Ridge Y-12 Plant, December 8, 1994, Sect. C, p. 9. 


\section{Appendix A: CAVIS SENSOR BUS PROTOCOL AND COMMANDS}

\section{$\operatorname{Rev} 1.0$}

\section{COMMUNICATIONS PROTOCOL}

For the purpose of this document, a communications protocol is a technique for organizing bytes of binary data into a structured data packet for transmission and reception. Using a protocol provides the software mechanism for; detecting communication errors in addition to hardware error detection, for tagging data packets as intended for a particular receiver, and transmitting raw data (e.g., such as might be read from $\mathrm{A} / \mathrm{D}$ converters). This results in significantly reducing the risk of the raw data causing decoding errors at the receiving end. First, a receiving sensor concentrator must determine when a valid data packet is received. If a valid packet is received, data in the packet is tested to determine the intended receiver. The communications protocol has an ID byte reserved for the purpose of identifying the intended receiver of the packet. The concentrator's hardware address switch is read and compared to the ID contained in the received data packet. If the two ID's compare then the command contained in the packet is executed. If the two ID's do not agree, the command and the packet are ignored. If the various $A / D$ readings taken by the sensor concentrator are transmitted back to the SPCS without the use of a protocol, there is a high probability that communications errors will occur. The communications protocol places the ID byte in a predetermined position in the transmitted packet and frames the data bytes with predetermined values. This makes it possible for the receiving end to perform tests on packets to greatly reduce the probability of false decoding.

\section{DESCRIPTION OF THE COMMUNICATIONS PROTOCOL}

The communications protocol for the CAVIS Interface System is described below. Under this protocol, all data and command bytes will be transmitted in a packet format using the following structure:

The first, second and third bytes will always be the ASCII code for Start of Text referred to by the mnemonic STX (packet header). These three bytes serve to form the head of the data packet and are used to obtain sync with packet transmission.

The fourth byte transmitted is the number of bytes in the packet represented as an eight bit binary number. This byte field is referred to by the mnemonic NCHAR. and ranges from 10 to 255 . This field will never be less than 10 since the packet overhead is 9 bytes and one data byte is required to transmit a command. The byte count is the actual number of bytes transmitted including the packet header STX,STX,STX and CKSUM.

The fifth byte is designated as the identification byte and reflects the intended receiver of the packet. This byte is referred to by the mnemonic $I D$. The $I D$ byte value of zero is reserved as the data collection computer's $I D$. The $I D$ of 255 is reserved for special use and should not be used as a sensor concentrator address. The sensor concentrator $I D$ 's should be in the range of 1 to 240 .

The next field of the packet is the data field where the transmitted data sent by the sensor concentrator starts or the command sent by the data collection computer is stored. This is a variable length field and can range from 1 to 246 bytes long. The packet protocol 
has an overhead of 9 bytes leaving a maximum of 246 bytes available for data or commands. In the case of transmitting multiple commands, the ordering of the commands in the data field determines the priority of execution. (i.e., the commands are executed in the order they are received starting with the first data byte.)

Byte positions (NCHAR - 3), (NCHAR - 2) and (NCHAR - 1) are always the ASCII code for End of Text referred to by the mnemonic ETX (packet tail).

Byte position NCHAR is the check sum of all the previous bytes and is referred to by the mnemonic CKSUM. The check sum is calculated by simply adding each byte to an initial 8 bit value of zero ignoring overflows. The receiving end performs the identical operation on the received packet and compares the answer to the transmitted check sum.

A graphical representation of a packet is shown below with the first byte starting at the left.

\begin{tabular}{|l|l|l|l|l|l|l|l|l|l|}
\hline STX & STX & STX & NCHAR & ID & DATA $_{1} \ldots$ DATA $_{(\text {NCHAR - 4) }}$ & ETX & ETX & ETX & CKSUM \\
\hline
\end{tabular}

The following steps are applied to test a received data packet for errors. Steps one and two are mandatory. Step three is used for an even higher level of confidence that a good packet was received without data corruption.

1. Test that the number of bytes received in the packet equal the value located in NCHAR.

2. Calculate the check sum of the received data packet by doing a modulo 256 sum of all the bytes in the packet except the last byte (CKSUM). This sum must equal the CKSUM value in the last byte if the data packet is to be considered a good packet.

3. To help insure against the unlikely occurrence of a compounded data corruption which results in a correct check sum calculation even through an error has occurred, test that the data frame is correct. The first, second and third bytes in the packet must be equal to STX and the bytes at NCHAR-1, NCHAR-2 and NCHAR-3 must be equal to ETX. 


\section{COMMUNICATIONS ALGORITHM USED BY SENSOR CONCENTRATOR}

The Serial Communications Interface of the sensor concentrator's micro controller generates an interrupt each time a character is received. The interrupt service routine that processes the interrupt collects the incoming characters, looks for the packet head and marks the packet buffer at the end of a packet. Tests not performed by this routine must be performed by the function that processes the received packets. Since the service routine only starts packets with three STX characters at the beginning and checks the range of NCHAR, the packet processing routine does not need to perform these tests. The following word diagram describes the interrupt service routine process.

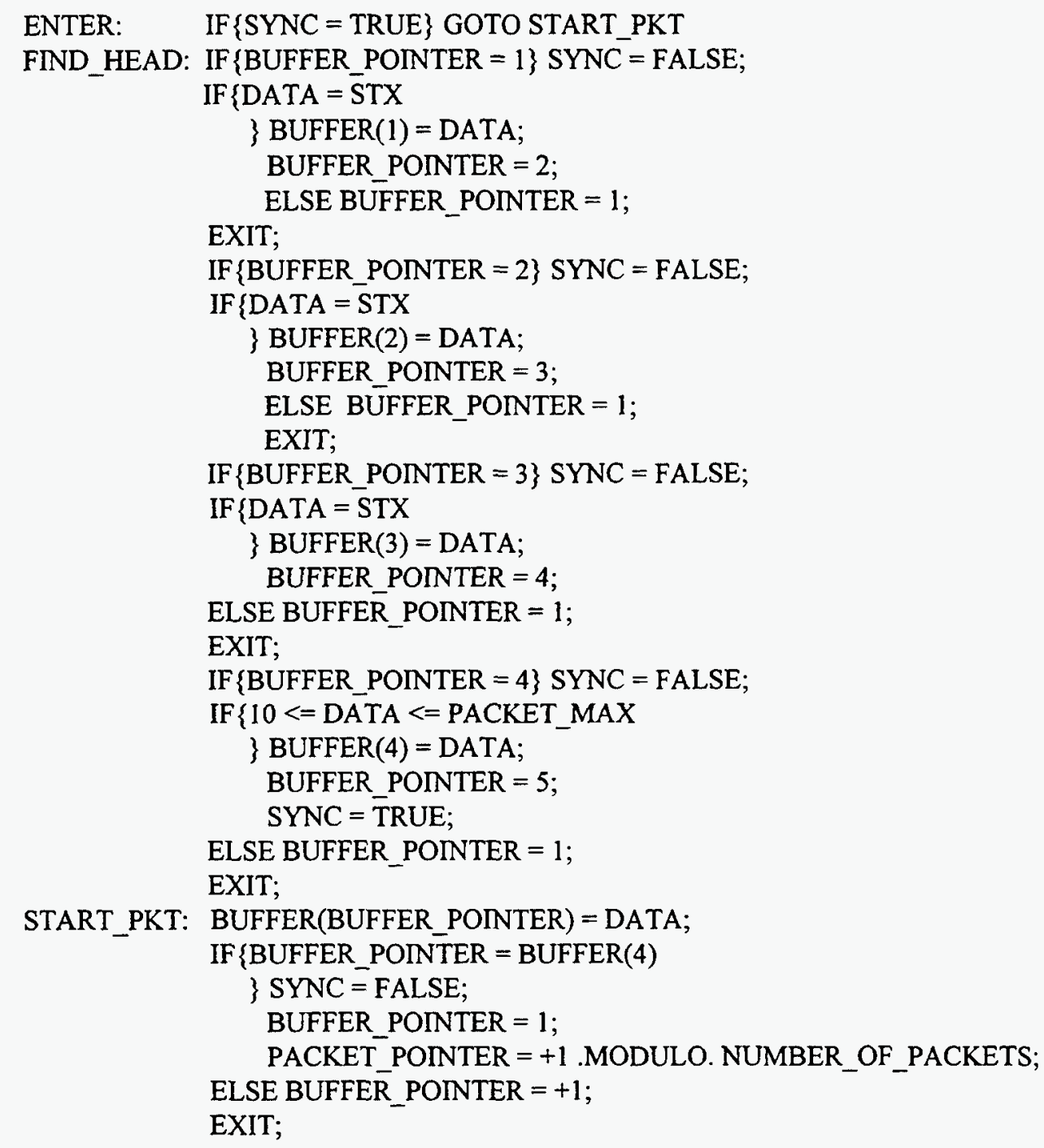

The sensor concentrator message processor checks for the presents of a new packet in the packet buffer. If a new packet is detected, the packet is tested to verify that it is valid. The interrupt routine performed the packet header test and verified the range of NCHAR so these two tests may be bypassed. The message processor first verifies that the packet tail contains three ETX characters. If the packet tail test is successful, the check sum of the packet is calculated and compared to the CKSUM field. If the CKSUM field and the calculated check sum agree, the 
packet is assumed to be good. If a packet test fails, the packet is zeroed and the message processor exited. Once a valid packet is detected, the packet ID field is compared to the local hardware address to determine if this node is the intended receiver. If this is not the intended receiver, the packet is zeroed and the message processor exited. If this node is the intended receiver, the command in the next data field after the ID is executed.

The following word diagram describes the sensor concentrator message processor:

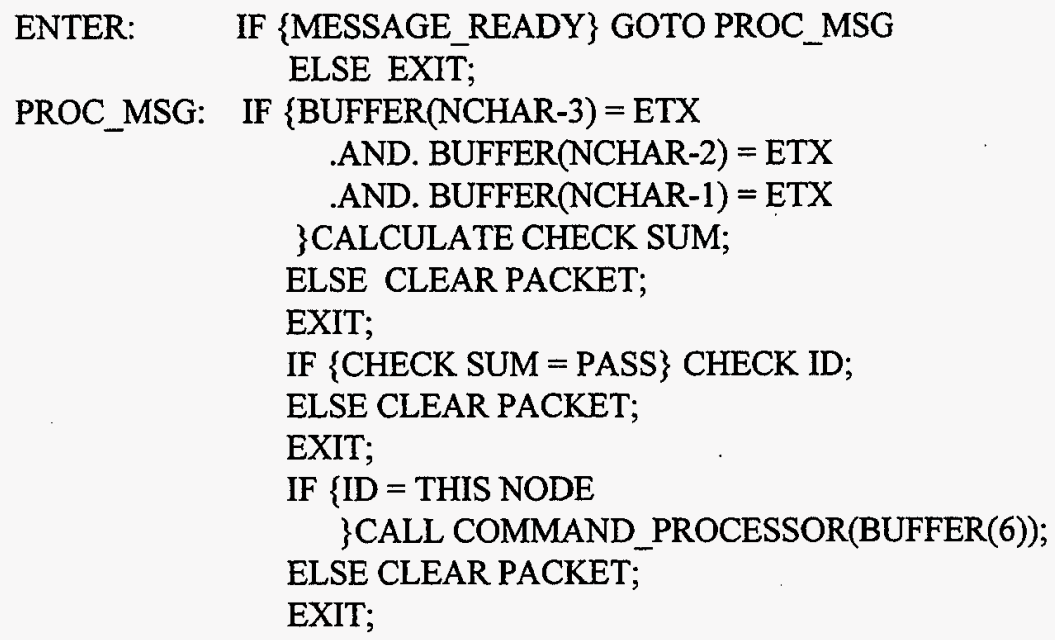

\section{COMMANDS TO SENSOR CONCENTRATOR}

The following commands are defined for the sensor concentrator:

REPORT -- Defined as decimal 01. Causes the addressed sensor concentrator to report the most recently taken readings for all the sensors.

REPORT_STATUS -- Defined as decimal 02. Causes the addressed sensor concentrator to report status information about calibration results, communications errors and other errors.

ARE_YOU_THERE -- Defined as decimal 03. Causes the addressed sensor concentrator to reply with a short packet containing only that concentrator's address.

RUN_CALIBRATION -- Defined as decimal 04. Causes the addressed sensor concentrator to run pre-defined calibration tests.

RESTART -- Defined as decimal 05. Causes the addressed sensor concentrator software to restart initializing all variables and data. 


\section{SENSOR CONCENTRATOR REPORT}

The communications packet returned by a sensor concentrator that has received the REPORT command is defined for the CAVIS demonstration module as follows:

Bytes 1, 2 and $3=$ STX

Byte 4 = NCHAR

Byte $5=\mathrm{ID}$ address of intended receiver

Byte $6=$ ID address of sensor concentrator that originated data

Bytes 7 and $8=$ Signed 12 bit reading for weight sensor 1 (MSB is byte 7)

Bytes 9 and $10=$ Signed 12 bit reading for weight sensor 2 (MSB is byte 9)

Bytes 11 and $12=$ Signed 12 bit reading for weight sensor 3 (MSB is byte 11)

Bytes 13 and $14=$ Signed 12 bit reading for weight sensor 4 (MSB is byte 13)

Bytes 15 and $16=$ Signed 12 bit reading for weight sensor 5 (MSB is byte 15)

Byte $17=$ Unsigned 8 bit reading for temperature sensor 1

Byte $18=$ Unsigned 8 bit reading for temperature sensor 2

Byte $19=$ Unsigned 8 bit reading for temperature sensor 3

Byte $20=$ Unsigned 8 bit reading for temperature sensor 4

Byte $21=$ Unsigned 8 bit reading for temperature sensor 5

Bytes 22 and $23=$ Signed 12 bit reading for gross gamma detector 1 (MSB is byte 22)

Bytes 24 and 25 = Signed 12 bit reading for gross gamma detector 2 (MSB is byte 24)

Bytes 26 and $27=$ Signed 12 bit reading for gross gamma detector 3 (MSB is byte 26)

Bytes 28 and $29=$ Signed 12 bit reading for gross gamma detector 4 (MSB is byte 28)

Bytes 30 and $31=$ Signed 12 bit reading for gross gamma detector 5 (MSB is byte 30)

Byte 32 and 33 = Unsigned 16 bit count for cps gamma detector 1 (MSB is byte 32)

Byte 34 and 35 = Unsigned 16 bit count for cps gamma detector 2 (MSB is byte 34 )

Byte 36 and $37=$ Unsigned 16 bit count for cps gamma detector 3 (MSB is byte 36 )

Byte 38 and $39=$ Unsigned 16 bit count for cps gamma detector 4 (MSB is byte 38 )

Byte 40 and $41=$ Unsigned 16 bit count for cps gamma detector 5 (MSB is byte 40 )

Bytes 42,43 and $44=$ ETX

Byte $45=$ CKSUM

The following " $\mathrm{C}$ " Language structure type is defined for the CAVIS demonstration software. This structure type is used in both the sensor concentrator and the data collection computer software to define memory storage for sensor data. In the sensor concentrator, only one storage structure is necessary, i.e.:

sensorDataType module;

In the data collection computer, one storage structure is defined for each sensor concentrator module in the system, i.e.

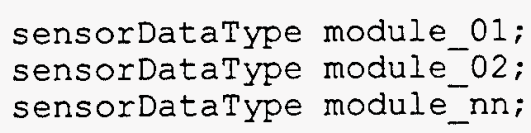




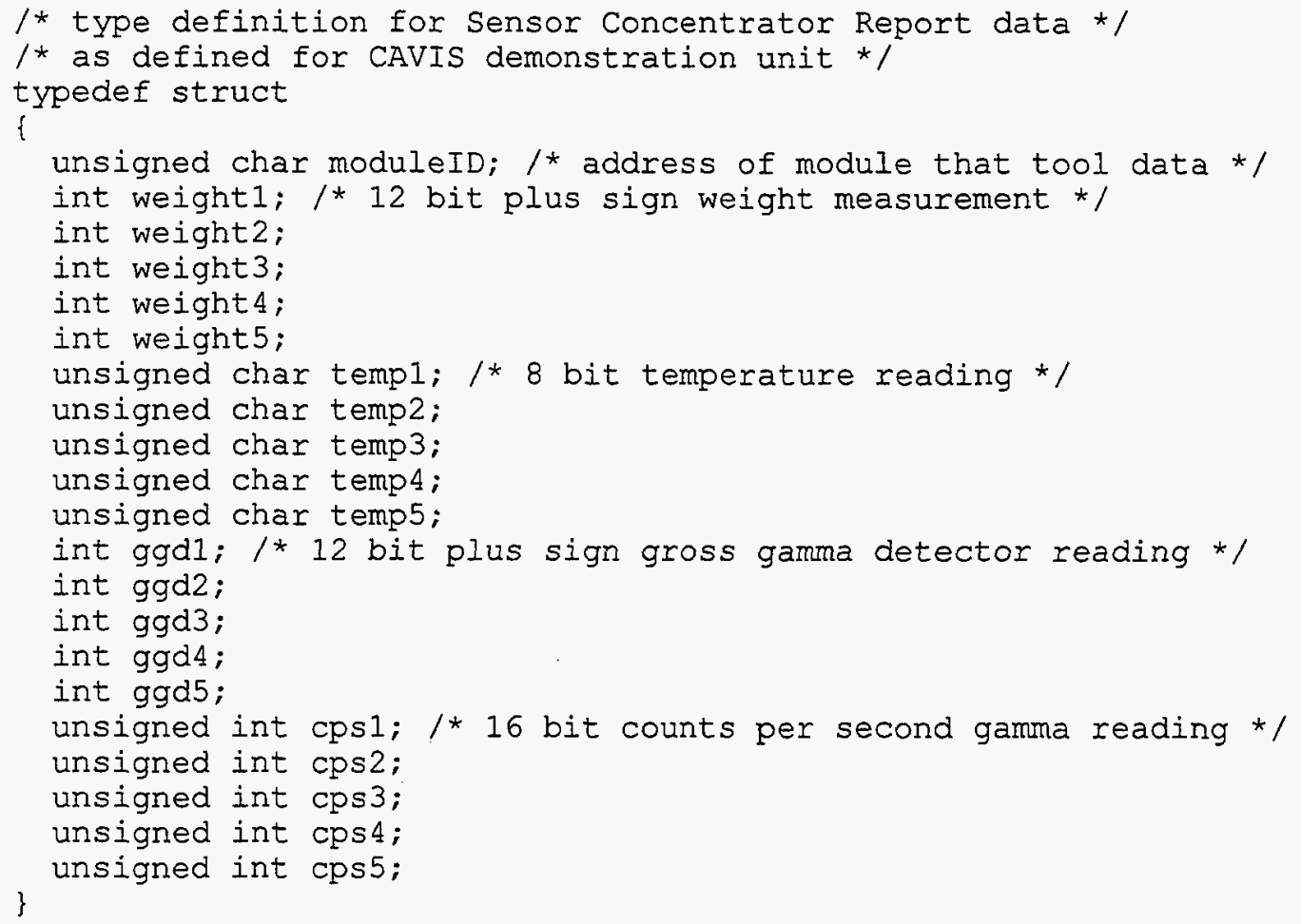




\section{INTERNAL DISTRIBUTION}

$\begin{array}{ll}1-5 & \text { R. N. Nodine } \\ 6-10 & \text { R. Lenarduzzi } \\ 11 & \text { G. T. Alley } \\ 12 & \text { D. A. Clayton } \\ 13 & \text { R. G. Gilliland } \\ 14 & \text { R. A. Hess } \\ 15 & \text { J. M. Jansen } \\ 16 & \text { D. W. McDonald } \\ 17 & \text { D. E. McMillan } \\ 18 & \text { G. N. Miller } \\ 19 & \text { G. S. Rao } \\ 20 & \text { C. W. Sohns } \\ 21 & \text { K. S. Weaver } \\ 22 & \text { J. D. White } \\ 23 & \text { J. A. Williams } \\ 24-25 & \text { Central Research Library } \\ 26-27 & \text { Laboratory Records } \\ 28 & \text { Laboratory Records-Record Copy } \\ 29 & \text { ORNL Patent Section } \\ 30 & \text { I\&C Division Publications Office }\end{array}$

EXTERNAL DISTRIBUTION

None 
M98054266

Report Number (14) ORNL/TM--13411

Publ. Date (11) 199706

Sponsor Code (18) DOE, XF

UC Category (19) UC-900, DOE/ER

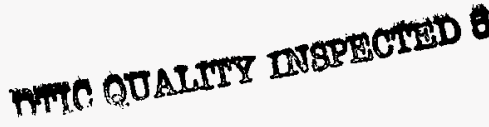

19990720098

DOE 